\title{
Disaggregating High-resolution Gas Metering Data Using Pattern Recognition
}

\author{
Ammar Alzaatreh*, Lamine Mahdjoubi, Bill Gething, Francisco Sierra
}

Centre for Architecture and Built Environment Research (CABER), Faculty of Environment and Technology, University of the West of England, BS16 1QY, Bristol, UK

* corresponding author: ammar.alzaatreh@uwe.ac.uk

\begin{abstract}
Growing concern about the scale and extent of the gap between predicted and actual energy performance of new and retrofitted UK homes has led to a surge in the development of new tools and technologies trying to address the problem. A vital aspect of this work is to improve ease and accuracy of measuring in-use performance to better understand the extent of the gap and diagnose its causes. Existing approaches range from low cost but basic assessments allowing very limited diagnosis, to intensively instrumented experiments that provide detail but are expensive and highly disruptive, typically requiring the installation of specialist monitoring equipment and often vacating the house for several days. A key challenge in reducing the cost and difficulty of complex methods in occupied houses is to disaggregate space heating energy from that used for other uses without installing specialist monitoring equipment. This paper presents a low cost, non-invasive approach for doing so for a typical occupied UK home where space heating, hot water and cooking are provided by gas. The method, using dynamic pattern matching of total gas consumption measurements, typical of those provided by a smart meter, was tested by applying it to two occupied houses in the UK. The findings revealed that this method was successful in detecting heating patterns in the data and filtering out coinciding use.
\end{abstract}

Keywords: gas disaggregation, smart meters, gas heating, Building Physics, dynamic models

\section{Introduction}

The building sector holds great potential for saving energy and reducing $\mathrm{CO}_{2}$ emissions, due to its large share of global energy use and the relative cost-effectiveness of energy saving measures [1]. In order to tap into this potential, policy makers across the world are imposing increasingly stringent requirements on building energy performance and introducing schemes to incentivise energy saving measures. However, the actual savings realised as a result of implementing those policies have repeatedly been shown to fall short of theoretical predictions [2]. As the scale and extent of this performance gap [3] has become clear, policy makers and the construction industry have become increasingly concerned that the shortfall could fundamentally impact the ability of the building sector to deliver its anticipated share of the national carbon reduction plan.

Addressing the performance gap requires methods to assess and understand the energy performance of existing buildings at scale, using methods that allow diagnosing the reasons for the shortfall, whether relating to physical or human factors. Such methods do exist, but as the existing building stock is largely inuse, the challenge is for these methods to be applicable to occupied buildings with minimal cost and disruption to occupants, but this issue has remained largely unsolved.

The main building performance measurement methods available are either based on intrusive heating experiments, such as the co-heating test and its various similar alternatives (e.g. QUB [4],ISABELE [5]); or on steady-state analysis of data recorded during normal operation and averaged over days or more, such as Energy Signature methods and a host of linear regression-based ones $[6,7,8,9]$, which try to quantify the relationship between heating energy and external-internal temperature difference and use it to characterise the energy efficiency of the building.

The first approach can be robust and provide accurate and detailed outputs, but is highly intrusive and expensive; it requires the building to be empty and unused for an extended period, along with extensive 
instrumentation [10]. The second approach, in contrast, is more practical and much less intrusive, but typical steady state models have long been deemed difficult to rely on for a detailed measurement that distinguishes between the underlying causes as required $[11,12,13,14]$. There are many reasons for this, of which the most important are statistical limitations regarding correlation of inputs, as well as the reliance on an assumption of consistent heating controls and occupant routines throughout the testing period. As such, some methods of this class produce outputs characterising the energy performance of the building as it is being used (e.g. the effective U-value, or the energy signature), which is no doubt an informative output, but it is not intended to make a distinction between the effects of system efficiency, operation, and envelope performance, and could well produce different ratings for physically identical buildings being used differently [7], which makes it of limited use for diagnosing underlying issues affecting measured performance.

The challenge is to have robust, detailed measurements of an occupied house, without disrupting occupancy. Dynamic analysis of energy and environment data has long been suggested as a solution to this problem $[11,15]$, and a growing body of research has demonstrated that it is potentially capable not only of characterising the energy performance in terms of its causes, but also of attributing the fabric performance to specific physical phenomena [16], in addition to having applications in optimal control [17], electric demand-side management [18], and other forms of intelligent applications. However, this comes at the expense of significantly increased instrumentation requirements compared to simpler options, of which the most important is heating energy measurement [19]. Unlike steady-state methods, for which the total energy consumption over long periods is usually sufficient, for dynamic analysis to be possible, it is imperative that a high frequency time series record of the energy output of the heating system is obtained, or, failing that, the energy used specifically for space heating. This is not as simple as making estimates by subtracting the average consumption of other appliances, or using correlations with weather or water consumption, as it has to provide the correct value at time steps traditionally less than 15 minutes. So far, dynamic analysis studies in the literature have largely tended to target testing facilities or highly instrumented buildings, like test boxes or office buildings with BMS systems installed $[20,19,21]$. When these types of studies are attempted in more common conditions, or in residential buildings, extra dedicated sensors have to be installed to monitor the energy used for heating $[22,23]$ among other variables.

The difficulty is most pronounced in the case of gas heating, particularly in residential buildings, where measuring the gas used by the heating system alone, or the heat output of the heating system, requires installing new meters, which are much more difficult and expensive to install than the other required sensors (e.g. temperature), and could require being installed within the piping itself. Even when the existing gas meter readings are sufficient, installing meter reading and logging equipment is problematic, due to the wide variety of meter types in use and the various limitations attached to them. In a recent survey of Building Performance Evaluation professionals in the UK [24], the consensus of respondents was that gas heating is the most difficult element to monitor in a domestic setting. As gas-fuelled heating supplies $42.2 \%$ of the total heating demand in Europe, and $80 \%$ of the total heating demand in the UK [25], this is a significant obstacle to any practical large-scale application of dynamic performance assessment methods, and the potential impact of addressing it is very significant.

In recent years, smart meter deployments have presented a great opportunity for these methods to become practical, by making the collection of meter readings easy and unintrusive. However, smart meter readings are still only a record of the total fuel usage of the building, which for natural gas typically includes domestic hot water and cooking in the UK, providing no immediate insight into the individual end use of heating, and certainly not the high frequency measurements of it required for a dynamic method. This challenge, often known as the disaggregation problem [26], or single-channel source separation [27], has been attacked in various ways in many contexts, but in cases similar to this one where a high frequency temporal sequence is required, dynamic pattern recognition has often been used with success [28]. Examples of this include the separation of readings from other types of smart meters measuring water use or electricity; however, no such method has been attempted for gas systems yet, where existing statistical models for disaggregation aim for separating sums [29], rather than high-resolution time series. 
Though the aim is similar here to the case for electric or water smart meters, the volume of use for different gas appliances is highly variable, and appliances usually control the flow with a mechanical valve subject to various temperature or even manual controls, which produces very different load patterns than those found in other types of metering. In addition, the effects of overlap in the case of combination boilers are more complicated, as gas consumption is not simply the sum of the use by two end uses, and needs to be estimated in other ways.

This paper presents a method for disaggregating the heating load out of the total recorded gas load by analysing activity in high frequency metering data, selecting candidate activity windows based on step changes in gas flow, and comparing them to a known instance of heating using Dynamic Time-Warping (DTW) as a similarity metric to detect probable patterns. The required input is simply gas measurements themselves, but weather and internal environment data, as well as information about the installed systems were used to validate the results in this paper. This approach was tested in a real-life case study, involving two occupied domestic buildings, with a similar building fabric, but contrasting gas energy use.

The paper is organised as follows; Section 2 reviews previous work, Section 3 describes the available data and case study. Section 4 discusses the features appearing in the usage profile and characteristics of the gas time series, Section 5 presents the proposed solution, and Section 6 presents results and evaluation, followed by discussion and conclusion.

\section{Previous Work}

Dynamic models of the thermal behaviour and energy performance of buildings first became a topic of research in the 1970s [31], and have since then advanced significantly [32, 33, 34], finding use in demand side management [18], smart thermostats [35], in-situ building performance measurement (e.g. ISABELE [5], QUB [4]), and more. There are many advantages to using dynamic models rather than steady state ones For building performance characterisation in general, such as the ability to assign energy use to different variables with confidence, but for an occupied building, the key advantage is that a dynamic model can identify the effects of operation (or other changes) directly, whereas a steady-state one would be blind to them, as the data is averaged over long time periods by necessity [11, 32].

Most dynamic models require, as a minimum, a high-frequency time series of internal temperature and heat flow into the building (produced by a heating system), in order to relate the amount of thermal energy expended to the degree of increase in temperature, and thus reason about thermal characteristics. To acquire the input heat data in an occupied building, the easiest option is to simply acquire access to an existing Building Management System (BMS) or instrumentation output from the heating system itself (e.g. [36]), but these are rarely available in a residential building. As a result, instruments for data collection in this sector have to be installed. This can take several forms varying in complexity and depending on the type of the heating system involved.

For gas boilers, installing a heat meter is perhaps the most preferable option, as demonstrated in [34] and [37]. This consists of a flow meter and a pair of temperature sensors on the supply and return pipes, which, combined, provide a measure of the thermal energy supplied to the building. However, installing a heat meter in an occupied domestic building is by no means a trivial task; it is often required to manipulate the insulation around the pipes, or in the case of more conventional in-line meters, the piping itself; and access to this pipework may not be easily available without significant disruption to the occupants, who could have to arrange to be in while work is carried out, have to clean cupboards to allow access to the pipework, and potentially suffer disturbance to the heating system. As a result, the commonly accepted alternative is to record the measurements of the existing gas meter, typically in volume units, and then convert them into energy. This is less direct and prone to error from several sources, such as the variation in fuel energy content and boiler efficiency; which are not likely to be very problematic, and meter reading inaccuracy; which is more likely to be so. Metering errors can result from many issues related to the metrological performance of diaphragm gas meters, such as inaccuracy at low flow rates, and gas volume changing with temperature [38]. Field tests of installed gas meters often find a number of issues with them [39] [40]. 
Nonetheless, as this method bypasses the requirement for installing new meters and reduces cost and difficulty considerably, it is a frequently taken option in many cases, particularly when heating is the only end use of natural gas in the building (e.g. [42], [43]). However, where gas is also used for domestic water heating or cooking, this method is problematic, as other uses have to be accepted as errors in the measurement of heating energy, or else resort has to be made to one of the earlier methods requiring added instrumentation. Thus, a method for disaggregating meter readings is likely to prove useful, at the very least by reducing the error margin back to that of the meter itself, rather than both the meter and presence of non-heating usage.

Disaggregating single point metered resource consumption by end use is a recurring problem in the literature. In the context of electrical consumption, this is known as Non-Intrusive (Appliance) Load Monitoring (NI(A)LM), and it has been an active field of research starting in the early 1980s. [44] Though early work in NILM made use of several measures specific to electric loads (e.g. using both voltage and current loads [45], reactive and active power [46], ..etc) or of very high frequency readings on the order of several $\mathrm{kHz}$ [46], more recent work has focused on using data obtained by measuring a single quantity (real power) at realistic intervals of 1 second or more, using methods mostly based on event detection and pattern recognition [28]. This type of data is presumed identical to the output of a smart meter should its frequency be high enough, but most deployed smart meters store and upload readings at a maximum frequency of one per 15 minutes. Some researchers have tackled the problem at this frequency or lower [47], but most choose to assume that readings will be recorded locally, as most smart meters are in fact capable of taking a reading every second or less, usually only for transmission to the local monitoring console or similar device. A review of the specifications of some of the most common models being deployed in the UK supports this assumption $[48,49]$.

Most methods employed in this class of problems rely on pattern recognition of appliance signatures, which are collected in dedicated experiments where the output of each appliance is recorded in isolation. With these signatures in hand, disaggregation is carried out by detecting switching events in the load graph, and then finding the sequence of appliance switching that is most likely to create the most matching graph [50]. This concept is widely applicable to problems other than electric loads, such as water metering [51, 52], but due to differences in the particular switching behaviour of the various systems, developed solutions are not usually portable to problems other than those for which they are intended.

Though methods developed to use smart meter-like readings have found use in different fields dealing with similar problems, the problem remains unaddressed for gas readings. Although statistical approaches to gas disaggregation have been developed [26]; they are intended only for disaggregating total volumes over some time period, rather than usage in the single time step, as required for dynamic models. Some hardware solutions to the problem have been introduced, in the form of extra sensors to be added to the metering infrastructure $[53,54]$, but of course solutions requiring production and installation of hardware are less desirable and do not solve the problem at a scalable level. For meter reading-based disaggregation, the only relevant published work to this problem so far is that of Bacher et al. [33], who, driven by the same aim of modelling the thermal behaviour of buildings without dedicated and intrusive sensor installation, manage to separate the total heat load of a district heated house in Denmark, recorded with a heat flow meter $(\mathrm{MJ} / \mathrm{h})$ at a resolution of 10 minutes, into space heating and water heating. Although this is not a gas system, the objective, being energy data of the heating system alone, is the same. In that work, the researchers differentiate heat load from water heating load by comparing periods of typical operation to a period without occupancy but with space heating operating as usual. They observe that the heat load in the house under study is slow-changing, of low relative magnitude, and is running continuously all day; whereas water heating takes the form of short, large spikes in the load graph, at magnitudes much higher than those of space heating. Based on that observation, they design a non-parametric estimator to filter out spikes in the total load graph, with the remaining, smooth usage assumed to be that of the heating system.

Though it may seem reasonable to expect similarity between the profile of heat load drawn from district heating and that of gas flowing into a local boiler, and though combi boilers do produce spikes when heating 
water for short periods; the main feature, that water heating causes distinctive spikes unlike those of space heating, does not seem to hold true for gas boilers as a general rule, and gas cooking is not a spike at all. A part of the issue is due to the different modes of operation, as it is quite rare for a UK house to be heated all day [55], and a load spike would be expected once heating starts.

It is thus apparent that disaggregation of gas metering high resolution time series by end use has yet to be demonstrated, and this paper attempts to address this gap.

\section{Data}

The data used in this study consists of time series of gas meter readings, taken at 1-min intervals in two 3storey, 3-bedroom, terraced houses in the south west of England (pictured in Figure 1), for a period of two months. Both houses are social housing properties built in 1971 using Wimpey No-fines Concrete, but later improved by fitting double glazed windows and installing external insulation. Combination (combi) natural gas boilers provide central heating and instantaneous domestic water for both houses, but only one of them uses gas for cooking. Table 1 provides further information, and Figure 2 shows the floor plan for both.

\begin{tabular}{|c|c|c|}
\hline & House 1 & House 2 \\
\hline Type & End terrace & Mid terrace \\
\hline Floor area & \multicolumn{2}{|c|}{$55 \mathrm{~m}^{2}$} \\
\hline External walls & \multicolumn{2}{|c|}{$\begin{array}{l}\text { No-fines concrete }(280 \mathrm{~mm})+\text { Paramount lining }(40 \mathrm{~mm}) \\
\text { Recently externally insulated with EPS }(110 \mathrm{~mm})\end{array}$} \\
\hline $\begin{array}{l}\text { Heat Loss Coefficient (SAP } \\
\text { Calculation) }\end{array}$ & \multicolumn{2}{|c|}{$720 \mathrm{WK}^{-1}$} \\
\hline Floor & \multicolumn{2}{|c|}{ Thermoplastic tiles, $9 \mathrm{~mm}$ screed, $100 \mathrm{~mm}$ concrete slab } \\
\hline Roof & \multicolumn{2}{|c|}{$\begin{array}{l}\text { Pitched roof with trussed rafters, } 100 \mathrm{~mm} \text { glass mineral wool on } \\
\text { ceiling }\end{array}$} \\
\hline Windows & \multicolumn{2}{|c|}{ Manual Double glazed PVC windows (closed most of the time) } \\
\hline \multicolumn{3}{|l|}{ Ventilation } \\
\hline - General & \multicolumn{2}{|c|}{ Windows + trickle vents (all rooms) } \\
\hline - Bathroom & \multicolumn{2}{|c|}{ Manual intermittent extract fan } \\
\hline - trickle vents & \multicolumn{2}{|c|}{ Above windows (closed) } \\
\hline - kitchen & Extract hood & - \\
\hline \multicolumn{3}{|l|}{ Hot water appliances } \\
\hline - Shower & Electric Instantaneous & Gas \\
\hline - Taps & \multicolumn{2}{|c|}{ Yes (gas) } \\
\hline - Bath & - & Yes (gas) \\
\hline Boiler model & Worcester Greenstar 25si & Vaillant EcoTECpro 24 \\
\hline - Type & Combi & Combi \\
\hline - Max output & 24kW & 24kW \\
\hline - Max DHW flow rate & $10.2 \mathrm{dm}^{3} / \mathrm{min}$ & $9.6 \mathrm{dm}^{3} / \mathrm{min}$ \\
\hline Gas cooker & Yes & No \\
\hline Heating control & $\begin{array}{l}\text { Single thermostat in the hall, } \\
\text { manually operated. TRVs in } \\
\text { rooms. Mechanical timer } \\
\text { control (unused). }\end{array}$ & $\begin{array}{l}\text { Single thermostat in the hall, } \\
\text { manually operated. TRVs in } \\
\text { rooms. Time programmer } \\
\text { (unused). }\end{array}$ \\
\hline Secondary heating & $\begin{array}{l}\text { Fan heater in main bedroom, } \\
1500 \mathrm{~W}\end{array}$ & $\begin{array}{l}\text { Portable electric heater, } \\
2000 \mathrm{~W}\end{array}$ \\
\hline Occupants & 2 & 1 \\
\hline
\end{tabular}




\section{Occupancy \\ Employed (late hours, evening and night) + Student \\ Employed (early morning start)}

Table 1: Information on studied houses

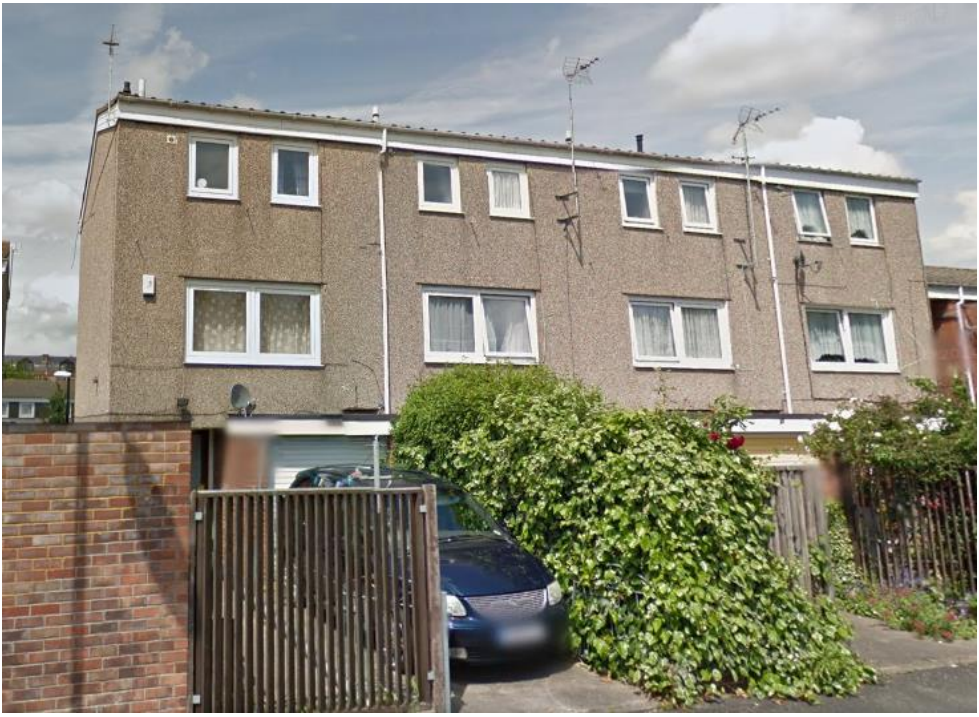

Figure 1: building with house 1

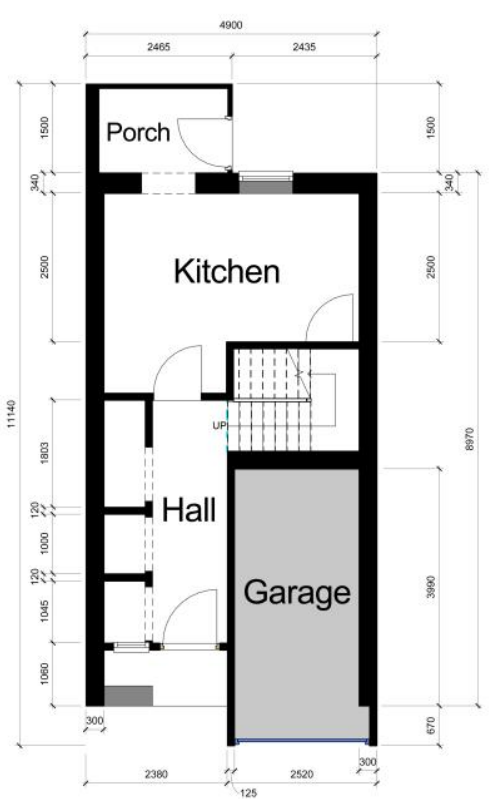

Ground Floor

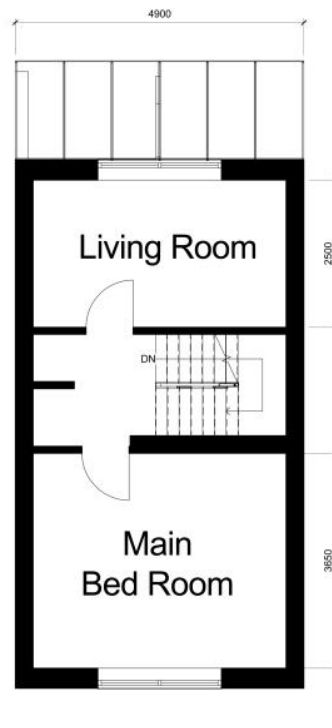

First Floor

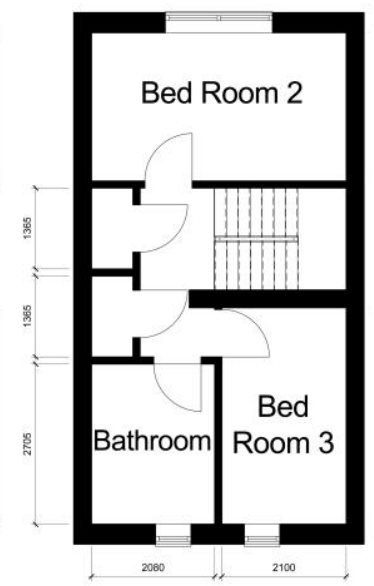

Second Floor

Figure 2: Layout of both houses

The owner (and main occupant) of house 1 is employed, working late hours usually starting late in the afternoon and returning at midnight, but they also work periodically overnight, leading to their hours of home activity mostly occurring around midnight or mornings. The other occupant is a student who is very often not present during the day, returning only in the evening. When the main occupant is not present, he tends to use heating for longer periods, and sometimes raise the thermostat set point. Heating seems to be manually switched on and off as needed, usually for one or two periods a day when the house is not empty, but the thermostat is rarely adjusted off its usual set point of $23^{\circ} \mathrm{C}$, and the time programmer is never used.

The occupier of house 2 is in full time employment, with work hours of 6-2 most of the week. They seem to keep heating running constantly all year, even when outside the house or asleep, and even during summer time (apart from rare hot spells). The set temperature of the thermostat is often changed in winter months, varying between $25-30^{\circ} \mathrm{C}$, but is left at $25^{\circ} \mathrm{C}$ in other times. 
Gas usage is recorded using magnetic detectors installed on the available gas meters (Figure 3), which count the number of digit revolutions in the front of the meter (corresponding to $0.01 \mathrm{~m}^{3}$ of consumption, or approximately $0.11 \mathrm{kWh}$ of energy), and send the total count (in the last minute) to the data logger, every minute. The resulting readings can be interpreted as the increase in meter reading in the last minute (volume), or else as a reading of gas flow, in units of $\mathrm{m}^{3} /$ minute and resolution of $0.01 \mathrm{~m}^{3}$, and can be converted into energy values by multiplying with the calorific value of the natural gas supply, which is given by the supplier as $39.4 \mathrm{MJ} / \mathrm{m}^{3}$. Figure 4 shows an example of the output for a 24-hour period in January 2017.
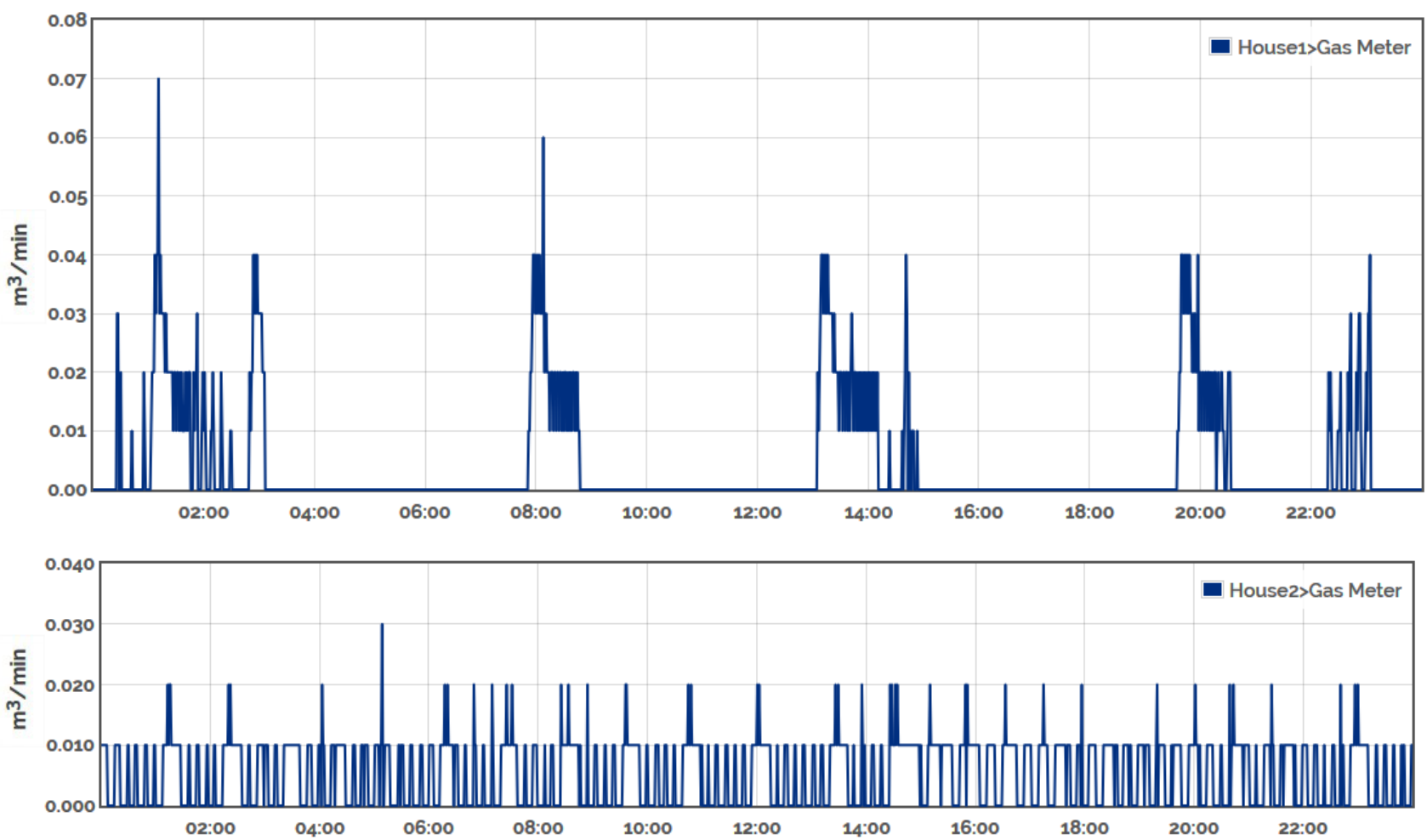

Figure 4: 24 hours of gas usage as recorded by the sensors in both houses

The monitoring frequency of gas usage, at 1 reading per minute, is chosen to capture the dynamic variation in gas use in as high a granularity as can be done while still being within the expected practical capabilities of a smart meter-based solution for large-scale deployment. That said, these readings are not truly representative of a smart meter reading in terms of accuracy, as the metrological performance of gas smart meters is expected to be superior to older types, simply by virtue of being newer, or potentially due to using newer technologies, such as ultrasonic (e.g. [40]) and thermal mass measurement, in some jurisdictions.

In the present study, the specifications for the available meters are given in Table 2, together with the calculated average flow rate in both houses. Both meters are equipped with temperature compensation elements, and have a magnetic pulse rate of $0.01 \mathrm{~m}^{3}$, meaning that the dials only trigger the magnetic detector at steps of 0.01 , despite the resolution of the meter reading being well below that. 


\begin{tabular}{|l|c|c|}
\hline Model & Itron U6 & Elster BK-G4 \\
\hline Max flow rate & $6 \mathrm{~m}^{3} / \mathrm{h}$ & $6 \mathrm{~m}^{3} / \mathrm{h}$ \\
\hline Min flow rate & $0.12 \mathrm{~m}^{3} / \mathrm{h}$ & $0.04 \mathrm{~m}^{3} / \mathrm{h}$ \\
\hline Cyclic volume & $2 \mathrm{dm}^{3}$ & $2 \mathrm{dm}^{3}$ \\
\hline Magnetic pulse rate & $0.01 \mathrm{~m}^{3}$ & $0.01 \mathrm{~m}^{3}$ \\
\hline Reading resolution & $0.0002 \mathrm{~m}^{3}$ & $0.0002 \mathrm{~m}^{3}$ \\
\hline $\begin{array}{l}\text { Average flow rate in house } \\
\text { (when in use) }\end{array}$ & $0.18 \mathrm{~m}^{3} / \mathrm{h}$ & $0.3 \mathrm{~m}^{3} / \mathrm{h}$ \\
\hline
\end{tabular}

Table 2: Gas meter specifications

In addition to gas meter readings, wireless air temperature sensors were placed in every room, and a weather monitoring station was installed on top of a nearby building to record local weather variables. This data was used for the purpose of development and validation, but is not part of the disaggregation method itself, and not required for application.

\section{Signature}

The basic premise of this work is that a heating system operates in a regular fashion, producing a temporal pattern of gas consumption sufficiently different from those of other systems that it can be relied upon to distinguish heating from other uses. Recognising and recording an appliance pattern could be done with a simple test, but unlike the electrical counterpart, there are usually only a few appliances using gas in a typical domestic building, and quite frequently, only one of them would be in use. This eliminates the need for dedicated experiments or sub-metering to record a signature, as the operation of different appliances can generally be discerned quickly by the analyst; however, some of these signatures can change in an analog way in response to human control or temperature variables, as with gas hobs for instance, which means defining a signature is not always simply a matter of extracting a 'clean' instance of it, nor of defining several states or stages.

For the present purpose it suffices to find the approximate signature(s) of the heating system, as all other usage will be discarded, and this signature is usually less difficult to work with. The operation of the modern gas boiler is largely dependent on a modulated firing cycle, switching between several levels of intake valve opening in response to the current temperature level of the fluid or a defined operation mode. This can produce several possible signatures, but the general shape is consistent between most boilers, composed of a high initial load as the boiler brings the heat up from rest, followed by modulated operation, which turns gas firing on and off, or high and low, as needed to preserve a certain target level of temperature. When the fluid temperature is already relatively high, the initial high load may not occur, and modulation may produce lower, or shorter pulses, depending on the boiler make and configuration. The result can take the shape of a train of slow on/off cycles, or a fast oscillation, or several stages of multi-level firing, but is in all cases a predictable effect of modulation.

Identifying the signature visually in the total load graph is not a difficult task. In most cases, heating operation will have a consistent daily or weekly pattern of occurrence, is absent in warmer days, and makes up the largest volume of gas use of all gas appliances, which makes it easily detectable on inspection. Any of these properties could possibly change depending on the type of system and the way it is operated, but save for some edge cases, this pattern is always likely to be the most prominent one. Figure 5 shows a 24 hour sample of the data in house 1 as an example, together with internal temperature data for validation. 

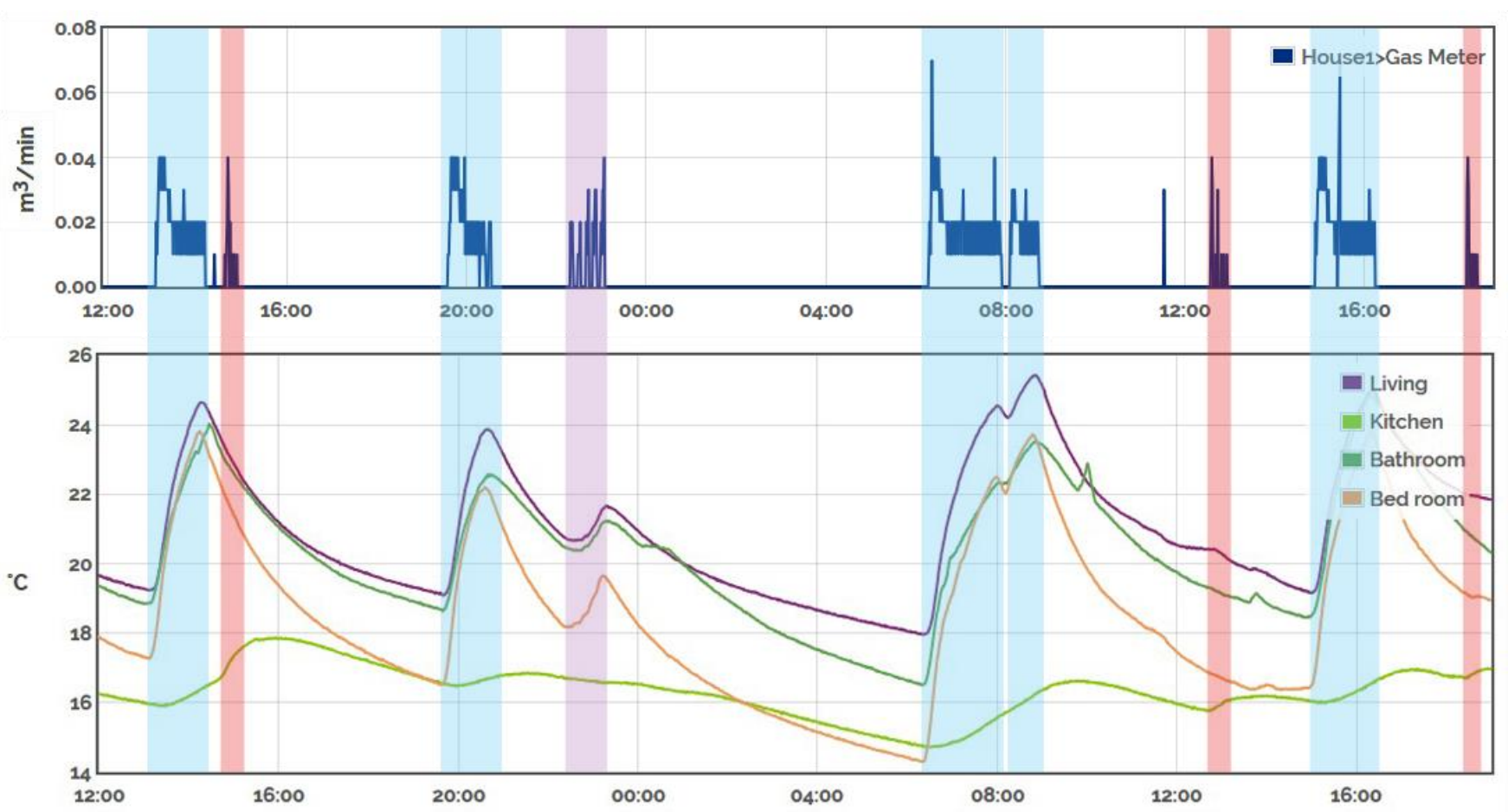

Figure 5: minutely gas usage graph for House 1 with patterns highlighted

Looking at Figure 5, it can be seen that three distinct patterns are immediately observable in the graph for House 1, with minor variations to each and some spikes. The most obvious candidate for heating is the first occurring pattern, being the most prominent and largest one, and matching the modulation effect expected of heating. This can easily be verified by observing temperature changes in the house at the same times. The pattern repeats five times in this period, and in all cases causes a sharp rise in the temperature in all rooms, which confirms that it is indeed caused by space heating. At approximately 13:30, a different pattern can be seen causing a rise in the temperature of the kitchen, but none anywhere else, which indicates that it belongs to gas cooking. The third pattern, at approximately 23:00, also causes a rise in temperature everywhere, but in a much slower manner than the first one. This is certainly also a result of heating, likely when less heating power is required. The remaining part is the irregular spikes, which are a typical result of water heating in a combi boiler.

Thus we can see that for House 1 , the signature takes the form of a sudden rise in gas flow, peaking at $0.04 \mathrm{~m}^{3} /$ minute $(26.2 \mathrm{~kW})$ and then falling again, over approximately 20 minutes. This is followed by a steady flow with a small oscillation, which continues at the same rate until heating is turned off.
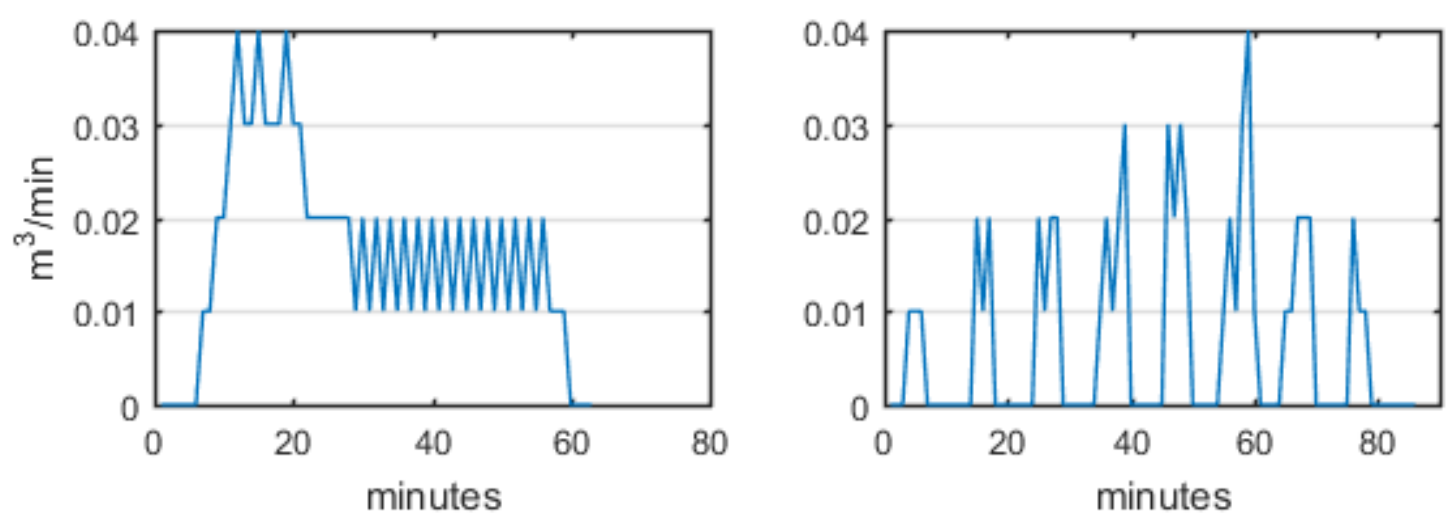

Figure 6: Space heating gas usage patterns in house 1

In House 2 heating is left running continuously, as this house is kept at a constant, high temperature all day long (rather unusual for a UK dwelling), but several occurrences when heating was turned off for several 
hours are available in the data. The observable signature during normal operation, as seen in Figure 7, is simply a series of rectangular pulses at a constant flow level but modulated width. Periodically, as temperature falls, this is replaced with a longer period of constant flow at the same level, with a spike or several spikes to a higher one (crossed with vertical lines in the figure).
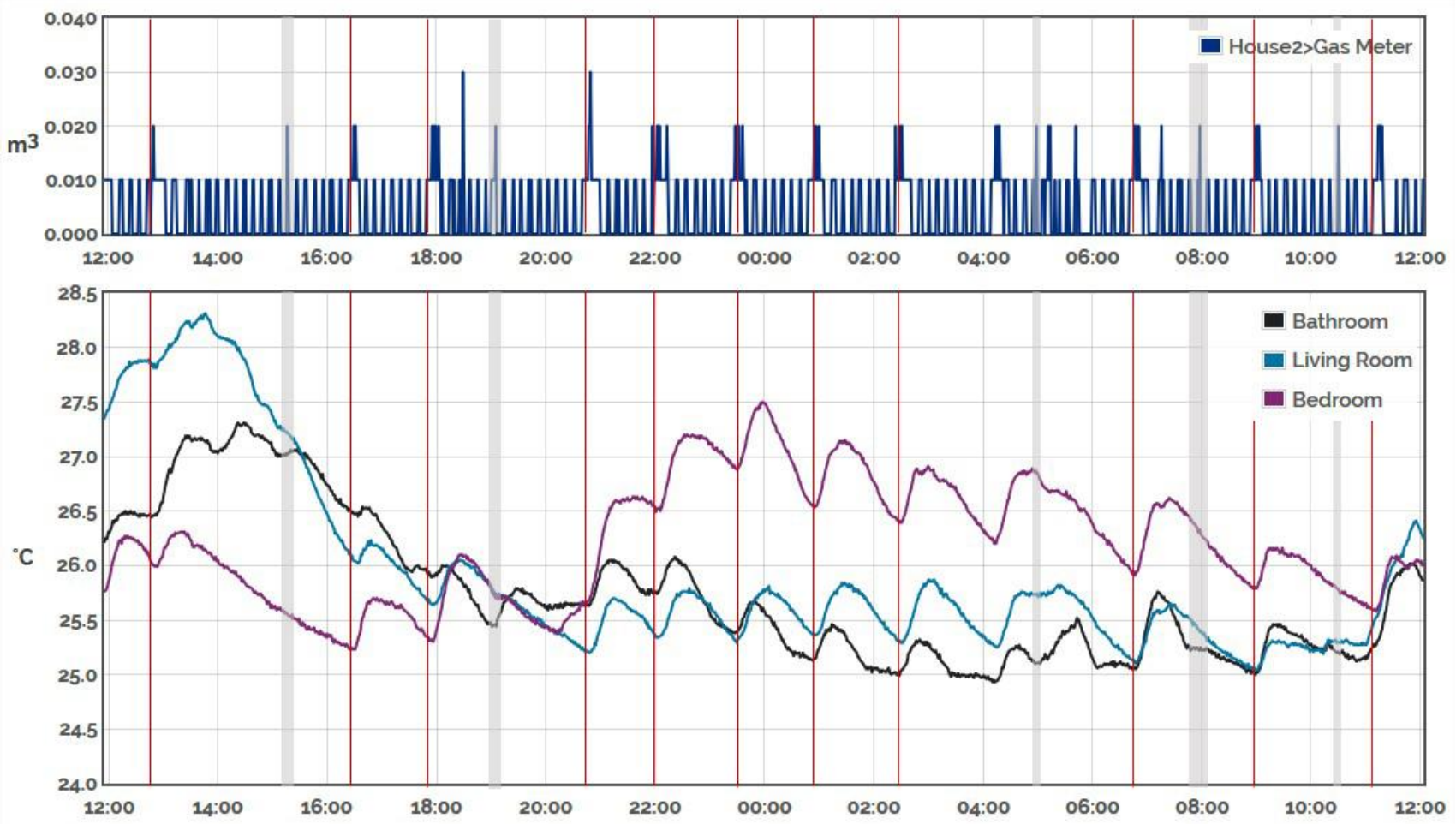

Figure 7: Gas usage patterns and temperature in House 2

As temperature rises clearly coincide with the periodic spikes, we can be confident that they are caused by heating. Spikes that do not occur during the longer flow period, like those observed at 15:10 and 18:20 in Figure 7 (highlighted), do not seem to coincide with a temperature rise, or else with a small rise in bathroom temperature, which likely indicates water heating for a tap load.

The remaining significant patterns that can be observed in the data are shown in figures 8 and 9 . Following the same reasoning process, these can be attributed with confidence to heating starting from rest and longer water heating loads, respectively.
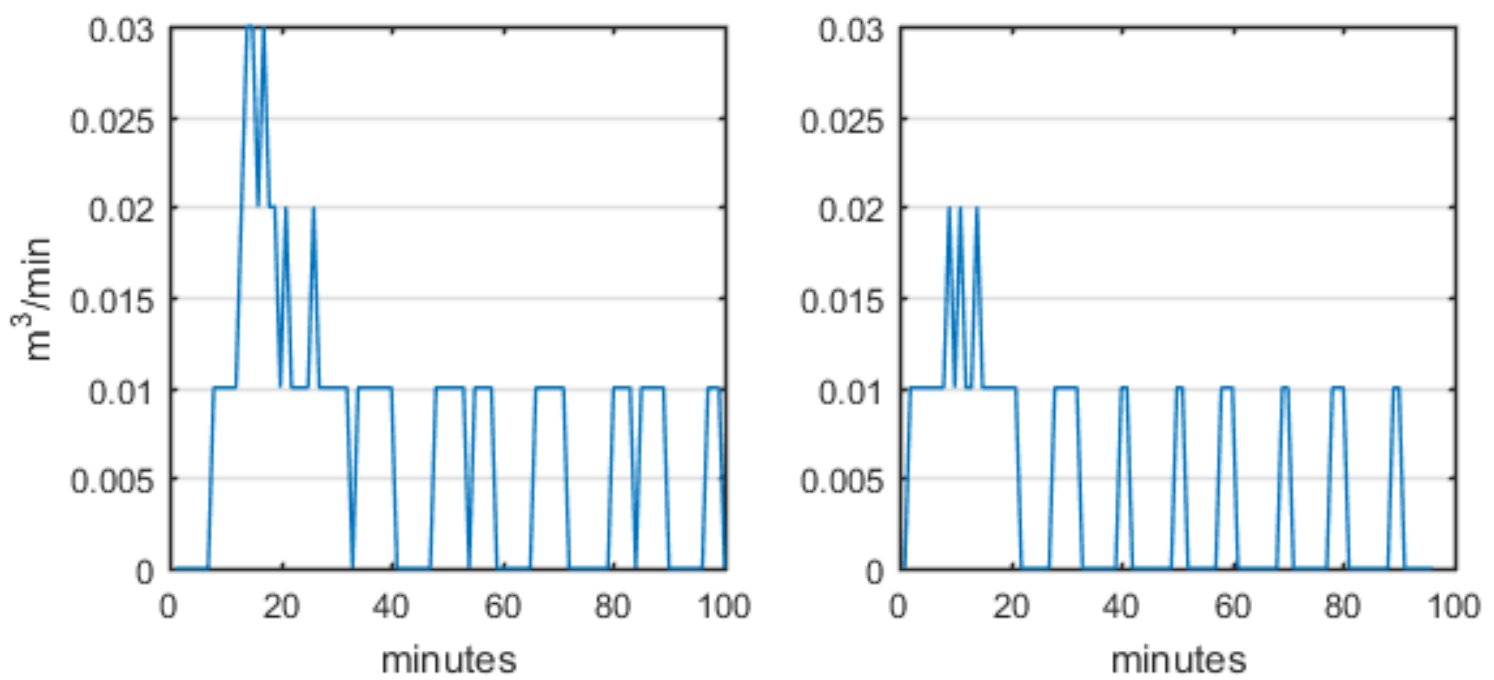

Figure 8: Heating gas usage patterns in House 2 


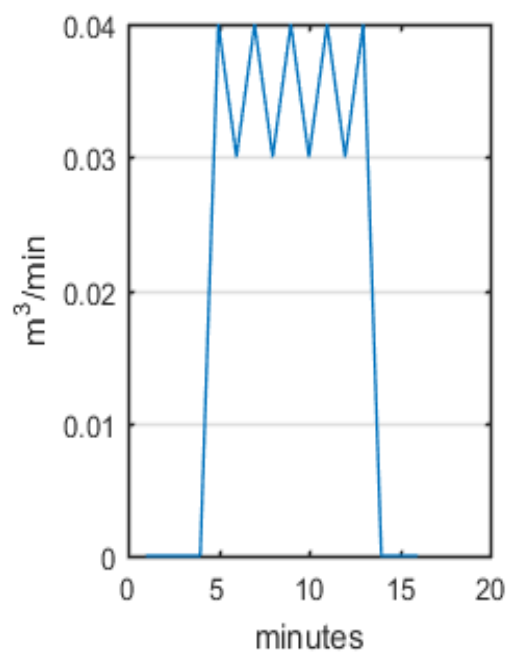

Figure 9: Gas usage pattern for extended water heating (e.g. running a bath) in House 2

It is evident that space heating signatures in both houses are discernible simply by observing the time series of measurements and identifying the most prominent pattern with a large volume and regular recurrence even in the absence of temperature data and information about the ratings or particular make and model of the boiler. In the process of conducting this study, the information used for validation was only consulted after signatures were initially identified. Expectation of the possible shapes for the profile of usage was guided by an understanding of the operation of the installed gas boilers, but the main reliable characteristic to look for was simply the large volume and regular recurrence.

\section{Method}

Based on the observations in the previous section, it seems to hold true that heating has a relatively consistent signature, which makes pattern recognition an obvious candidate for the task of detecting it among other uses.

The objective of the presented method is to detect periods when heating is on and filter out any nonheating usage, arriving at a record of the gas used for heating per minute. This is achieved by comparing the gas usage profile in all time windows of non-transient activity to all defined space heating signatures using a time series similarity metric, selecting windows that have a similarity above a certain threshold, and filtering them to remove any added usage that deviates too far from the average of all windows. A summary is shown in the flow chart in Figure 10.

In presenting this method we assume that the signature of the heating system has already been identified, whether by visual inspection, experimentation, or one of the other ways discussed in the previous section. 
Select a known heating period with no extra events, or obtain signature in other ways

\section{Filter and Threshold}

Smooth data, identify periods with non-transient activity by thresholding result

\section{Calculate Similarity}

Calculate smiliarity of each window to each space heating signature

Select similar windows

Select windows with high similarity

Reestimate Outliers

detect outlier values and re-estimate them

Figure 10: Flowchart of method

\subsection{Window detection}

The first step is to select candidate windows of activity, where a candidate window is one with continuous, sustained usage, beginning with a significantly large positive event (increased flow) and ending with a significantly large negative event (decreased flow). To select these windows, we begin by applying a smoothing filter to the time series, which creates a copy in which spikes and oscillations are removed, and all changes in the graph are smoothed out, simplifying it into coherent windows of operation (figure 11). Selection of candidate activity windows can then be carried out using the filtered version, simply by imposing a threshold value, and selecting any window crossing this threshold.

Starting with a series of gas readings $\left\{g_{t}\right\}$ taken at regular intervals, and the symmetrical moving average as a typical choice of smoothing filter, the series of smoothed readings $\left\{G_{t}\right\}$ can be calculated by

$$
G_{t}=\frac{\sum_{t=-(S-1) / 2}^{t=(S-1) / 2} g_{t}}{S}
$$

Where $S$ is the length of the averaging window.

Using the filtered output, window boundaries can be found by matching threshold crossing events in the smoothed series, for a certain threshold value of $T$.

$$
\delta_{t}= \begin{cases}0, & G_{t}<T \\ 1, & G_{t} \geq T\end{cases}
$$

$$
\Delta \delta_{t}=\delta_{t}-\delta_{t-1}
$$

Where $\left\{\Delta \delta_{t}\right\}$ is the time series of threshold-crossing (switching) events, indicating a rise in flow for positive events $\left(\Delta \delta_{t}=1\right)$, a fall for negative ones $\left(\Delta \delta_{t}=-1\right)$, or no change $\left(\Delta \delta_{t}=0\right)$. The smoothing of the series, if calibrated correctly, guarantees that these events will not be spikes or fast perturbations, but the start or end of sustained activity of a significant time length in most cases.

To define candidate activity windows, event times are split into positive and negative series. So for every $t_{n} \in t$, instances are distributed according to 


$$
t_{n} \in \begin{cases}E^{+}, & \Delta \delta_{t_{n}}=1 \\ E^{-}, & \Delta \delta_{t_{n}}=-1\end{cases}
$$

Assuming $g$ starts and ends at 0 (or is padded with zeros), both $E^{+}$and $E^{-}$will have the same length, meaning every rise above the threshold will be followed, eventually, by a fall below it. Thus candidate activity windows are simply subseries of the original gas readings series, between each two subsequent threshold-crossing events.

$$
W_{n}=\left\{g_{t}\right\}_{t}^{t}=E_{n}^{-}
$$

Arriving at this result by following these steps requires a choice of two parameters; the span of the moving average window $S$ and the threshold $T$, which have appropriate values different for each setup, and possibly for each signature. In this case they were tuned manually, but should the average length of heating activation in the house be known, they may be optimised to yield a match to the average candidate window length or some similar condition, but this is left for future work.
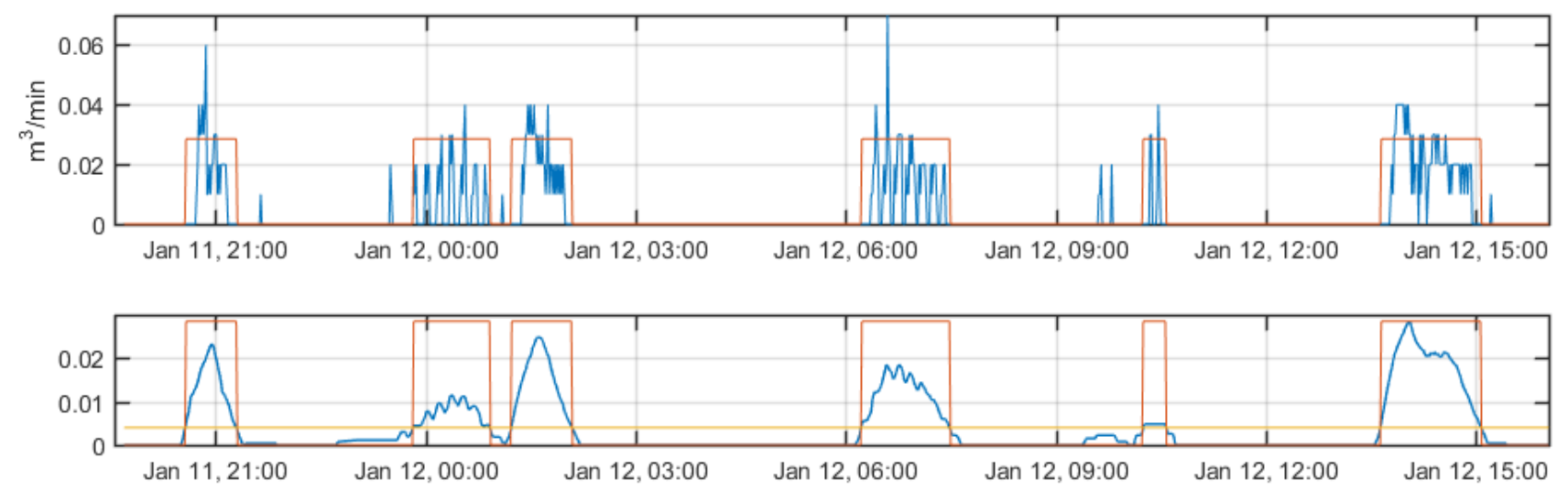

Figure 11: Filtering and Window Selection

\subsection{Similarity}

To distinguish heating from other uses, it suffices to detect the signature patterns. This is done by comparing identified windows of activity with the signature, using a standard similarity calculation algorithm. In the field of time series data analysis, particularly for the purposes of classification and clustering, Euclidean distance is widely used to measure the similarity of series, but its sensitivity to time axis distortions is a wellknown problem [56], wherein slight changes in the timing of a certain feature (e.g. peak) or the length of time between features within the series would affect the measured similarity highly. This has led to the introduction of Dynamic Time Warping distance (DTW) [57], an alternative similarity metric that has proven very highly successful in many fields, such as speech recognition [58] and bioinformatics [59, 60], and has been successfully applied to disaggregation problems similar to the present one, in both electricity [61] and water [51].

Dynamic Time Warping measures the 'distance' between two time series irrespective of any variations in the time dimension. The algorithm attempts to temporally stretch or compress parts of the compared signal until it finds the state with the shortest Euclidean distance to the standard one and the same length, which in this case allows the comparison of windows to signature irrespective of variations in length of firing stages or modulation times, and using only the data points themselves rather than any model of them. A side effect of this is that it sometimes inflates the similarity scores of windows containing very short or very simple profiles if the warping path is not optimised correctly [62]. This is not a serious issue however, as a properly selected threshold for window selection should cause most of these to be dropped in the detection stage. 
Figure 12 shows an example of DTW similarity computation. The best match between the detected candidate window and the signature is produced by stretching the detected signal to have the same length as the signature. It can be seen that the detected window seems to be an almost perfect match aside from an extra spike right before the modulation stage, which could be due to an overlapping use of gas, or due to heating itself, as will be discussed in the next subsection.
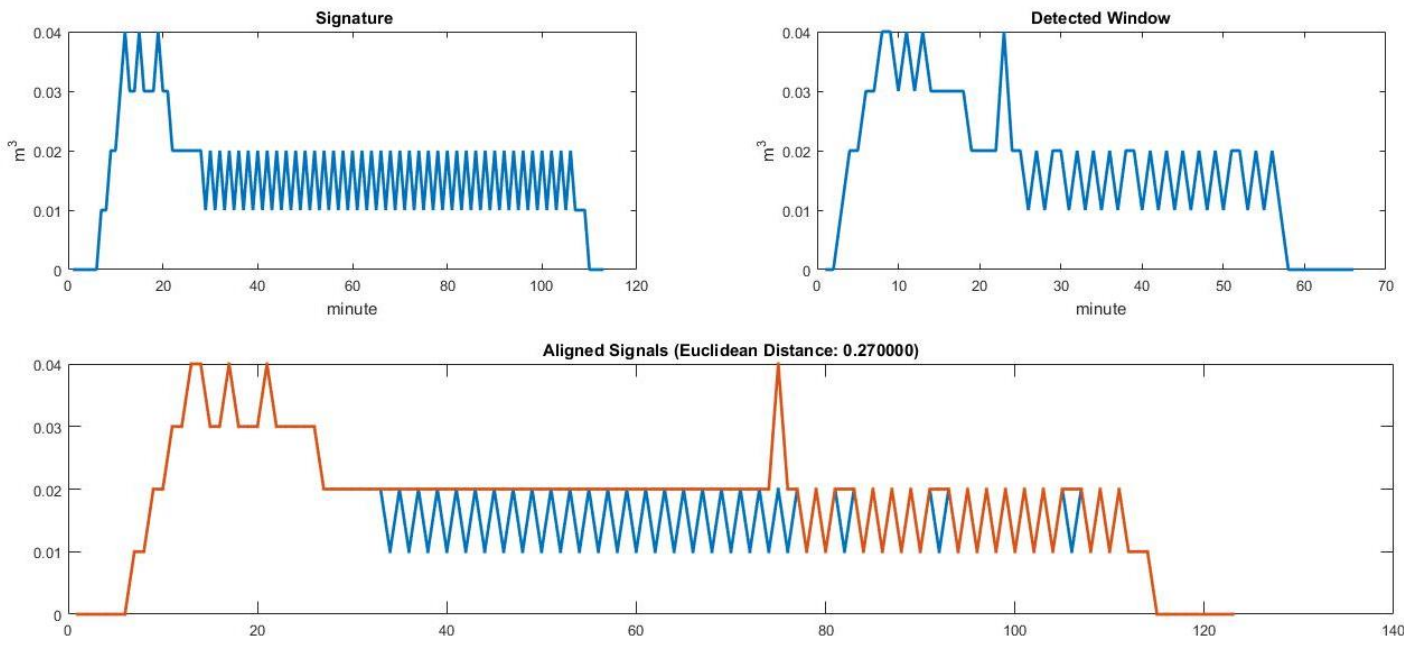

Figure 12: Alignment between signature and a detected window

The DTW distance of all selected windows to the signature is a measure of similarity, on which we can impose a threshold to select windows that are sufficiently similar to the signature to be judged as heating windows, with a simple condition

$$
H=\left\{W_{n} \mid D T W\left(W_{n}, \operatorname{Sig}\right) \leq \theta\right\}
$$

The set of heating activity windows includes every window $W_{n}$ in the set of all candidate windows $W$ where the DTW distance from a certain signature is less than a threshold $\theta$.

\subsection{Anomaly filtering}

Although heating is usually the dominant end use in terms of total time and volume, other uses may still coincide with a heating event in the same time window. In the absence of such events, the observable profile of heating in the time series matches the stored signatures quite closely, but variations and additional spikes do occur. The problem of filtering, thus, is to identify which differences from the signature are anomalies, resulting from non-heating usage rather than a natural variation in heating operation, and where this is determined to be the case, estimate the heating-only reading.

Anomaly detection is a common problem in data mining, with many existing solutions. Simply stated, the aim is to identify events that do not conform to expectation, whether the expectation is explicit (based on a known model, stochastic distribution, or known sequence of normal behaviour) or has to be inferred from the data itself [63]. The key insight relating to this case is that non-heating end uses are unlikely to highly correlate with heating events in time, and do not occur consistently at the same stage of heating operation with the same magnitude, which means the unwanted variations are simply those that occur infrequently.

In the context of the disaggregation problem, the signature is this 'expectation', often defined in terms of Markov chain models $[28,64]$, but in this case the signature is only a sample, rather than an accurate description or model of operation. This can be changed at this stage by training a model of detected heating windows, to serve as an expectation for anomaly detection. As the behaviour of heating is dynamic, relating to a stage of operation and values at several time steps in the past, and the set of possible values is limited, 
we can define this using a matrix of multi-step (reverse) transition probabilities, as an extension of the markov chain model. This approach is common in anomaly detection of discrete sequences [65].

Let $X$ denote the state at time $t$, where $X_{t} \in\{1,2,3, \ldots\}$ corresponding to the set of possible values of $g_{t}$, we can have matrix $P$ where

$$
\operatorname{Pr}\left(X_{t}=i \mid X_{t-1}=j, X_{t-2}=k, X_{t-3}=l\right)=P_{i, j, k, l}
$$

This matrix is easily populated by calculating the probability as the frequency of each value given the previous values, within the detected heating windows themselves, with points outside window boundaries assumed zero. This matrix must be calculated independently for each set of windows matching a certain signature, and then anomalies are simply any value where the probability is less than a certain threshold. When an anomaly is detected, a good estimate of the correct state at that point is simply the most probable state

$$
\hat{X}_{t}=\operatorname{argmax}_{x} \operatorname{Pr}\left(X_{t}=x \mid X_{t-1}, X_{t-2}, X_{t-3}\right)
$$

But for a combi boiler where only one use is activated at a time (space or water heating), this can simply be replaced with a value of zero.

This method of anomaly detection and re-estimation is rather generic and possibly computationally intensive. It depends on the possible readings being a discrete set, which, although not an obstacle to their application where better resolutions are available, wastes the information possibly contained in them. Other more elegant methods can be used here, particularly robust estimators, like that shown in [33], but for the present purpose this is deemed sufficiently effective.

\section{Results}

Applying this method to the available data yields a separation between space heating and other uses, as shown in figures 13 and 14. Figure 13 shows a day of results in House 1, the total usage graph (top), is split into a heating graph and a non-heating one by applying the methods described in the previous section. By visual inspection alone, it can be seen that all recognisable heating events have indeed been isolated, and several abnormal spikes in them have been smoothed out. Some features of interest are highlighted and numbered, with discussion to follow.
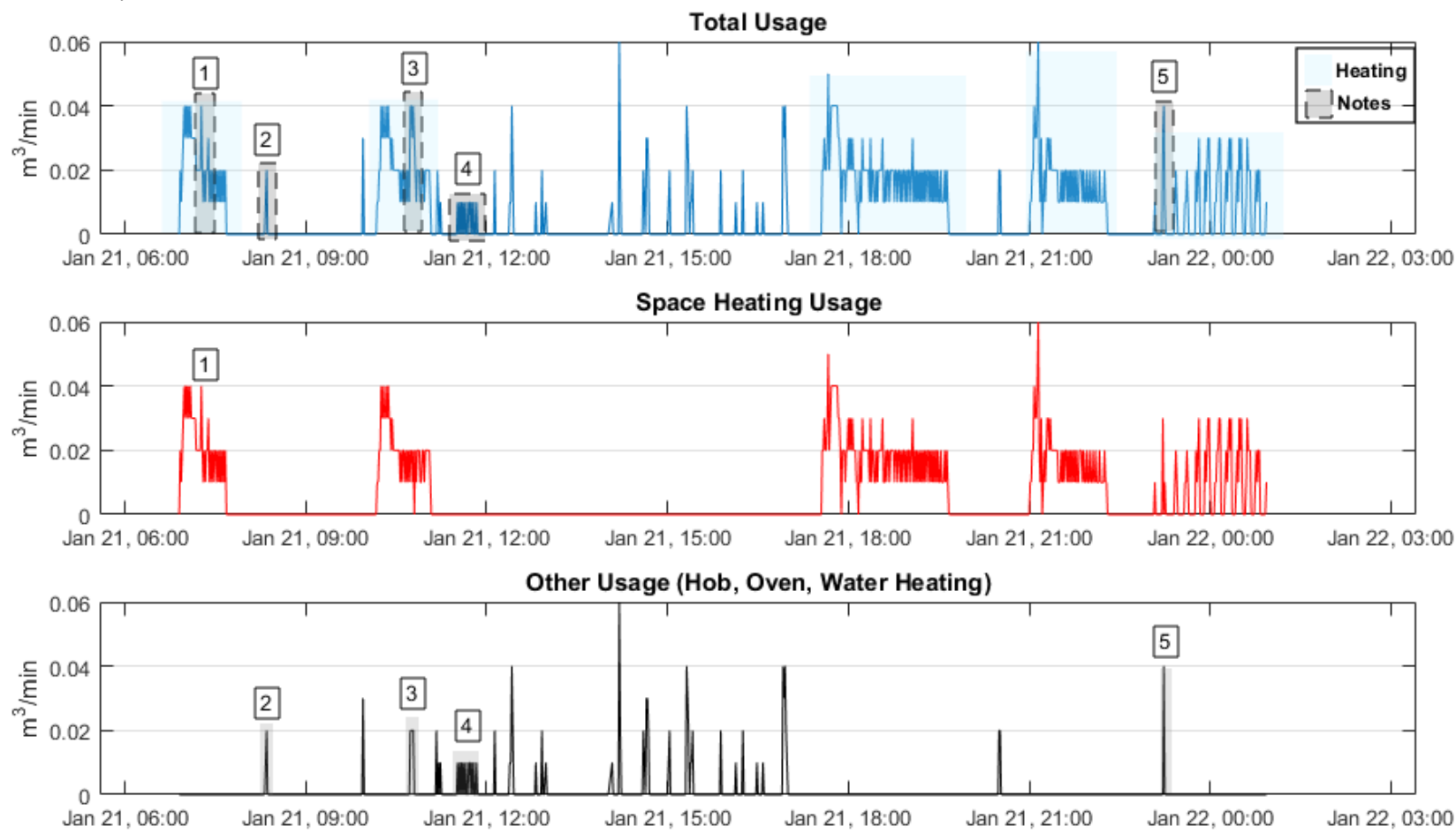

Figure 13: Demonstration of results in house 1 
Looking at the total usage graph, four instances of the main identified heating pattern can be discerned, and one of the second one. Starting at 07:00, the pattern is a match for heating, but it differs from the signature shown in Figure 6 by a spike in the middle (1). This spike has not been filtered out, as it happens often at this point in the heating cycle, and is thus calculated to be a variation in boiler operation. Near 08:30, marked by (2) is an isolated spike of gas usage, too short to be the result of space heating, and the same can be said of the following one, around 10am. Both are likely results of water heating, and are both filtered out. Right after, a heating cycle is detected, but in this case, after 30 minutes of uninterrupted heating, usage picks up unexpectedly (3); as this is irregular, the calculated probability of it being a variation in boiler operation is low and it is thus filtered out.

Shortly after 11:30 another pattern of usage appears (4). This pattern does not fit any heating signature and is thus removed into the other usage bin. Given its low volume, consistent level, and oscillation profile, this is likely to be gas oven operation. Thereafter, until 17:30, multiple spikes appear of different characteristics, but none of them is long enough to be detected as heating, and are thus removed. At 21:30 (5), a low-load heating pattern is detected, but a spike in usage a few minutes after it starts is judged irregular and removed. It is notable here that the anomaly filter does expect a spike of $0.03 \mathrm{~m}^{3} / \mathrm{min}$, as evident by the output, but the following increase up to $0.04 \mathrm{~m}^{3} / \mathrm{min}$ for 2 minutes was considered anomalous, showing that even if non-space heating results coincide with space heating, they can be detected.

In House 2 the results are easier to explain. Figure 14 shows a full day of results. It can be seen that space heating is running all day, causing a constantly oscillating gas flow, switching regularly back to a higher-use pattern, which appears three times between 08:00 and 09:00. Spikes that do not fit this profile have been filtered out. In particular, a period of extended use around 16:00 is removed as it does not fit the heating signature. This pattern is likely to be a result of extended water heating use, possibly caused by running a bath, as explained in section 3.
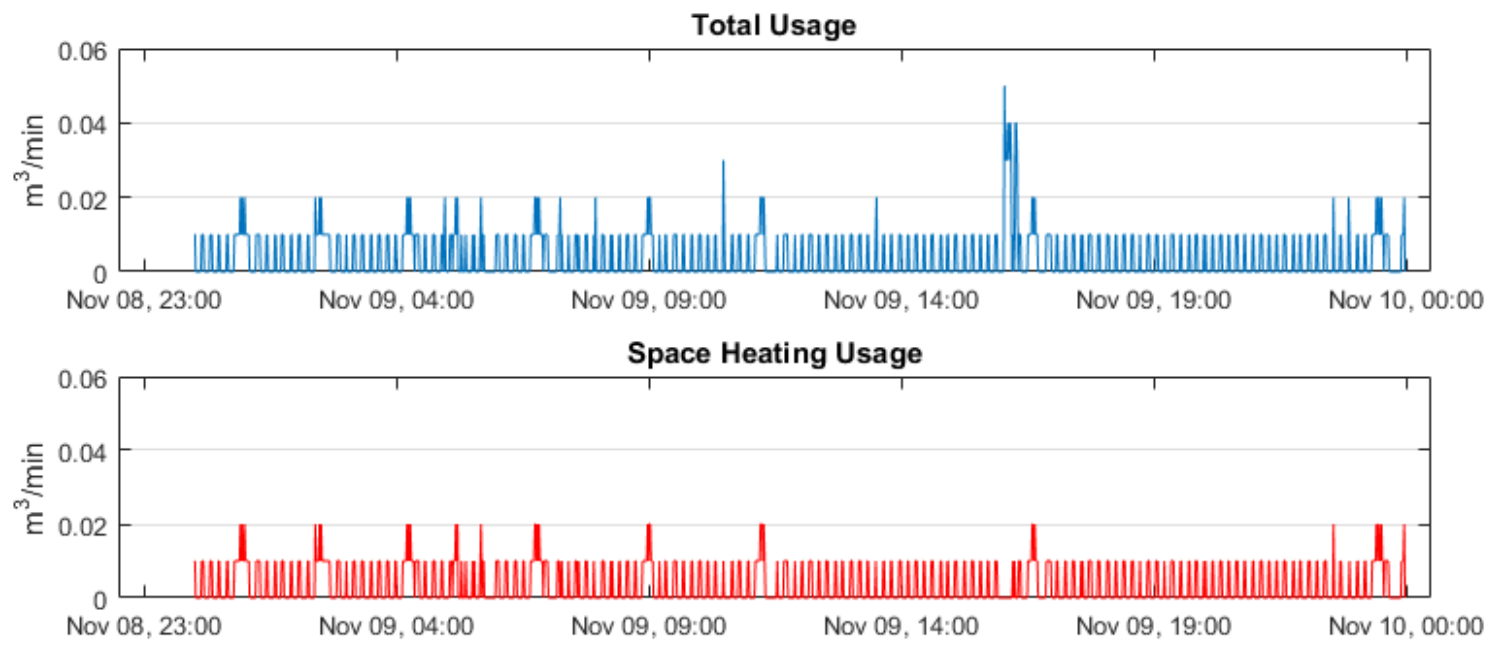

Water Heating Usage

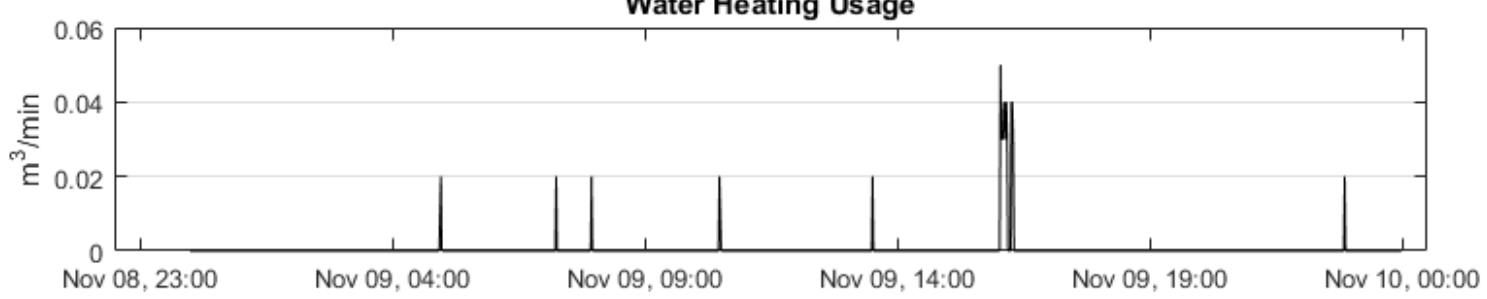

Figure 14: Demonstration of results in house 2 


\section{Validation}

Correct validation of these results requires a ground truth series, where gas usage for each of space heating, water heating, and gas cooking is measured independently. As the boilers in this case are both combi boilers, both water and space heating make use of a single gas intake pipe, and independent measurement is not possible to do externally. This, combined with occupancy restraints, makes correct direct validation difficult, and recourse has to be made to less ideal options. In order to validate the results, three tests were carried out. First, an experiment is carried out in House 2, in which water heating is triggered at set times to interrupt space heating, and the method is used to detect this. Second, a model predicting temperature rise in the houses based on gas usage is created, and then applied to show that disaggregated space heating gas usage, as given by the method, predicts temperature rises more accurately than the original total. Finally, the average daily profile of results is compared to the expected based on knowledge of occupant routines, showing that the results are consistent with expectations.

\subsection{Experimental validation}

The possibility of full experimental validation was constrained by occupancy considerations and boiler types, as explained above, but short experiments were possible in house 2 , where gas is used only for space and water heating.

In the first experiment, heating was turned off for 30 minutes, then started again from rest, and left running for 65 minutes with no water heating usage. The result, shown in figure [15], is consistent with the expected pattern found in section 4.

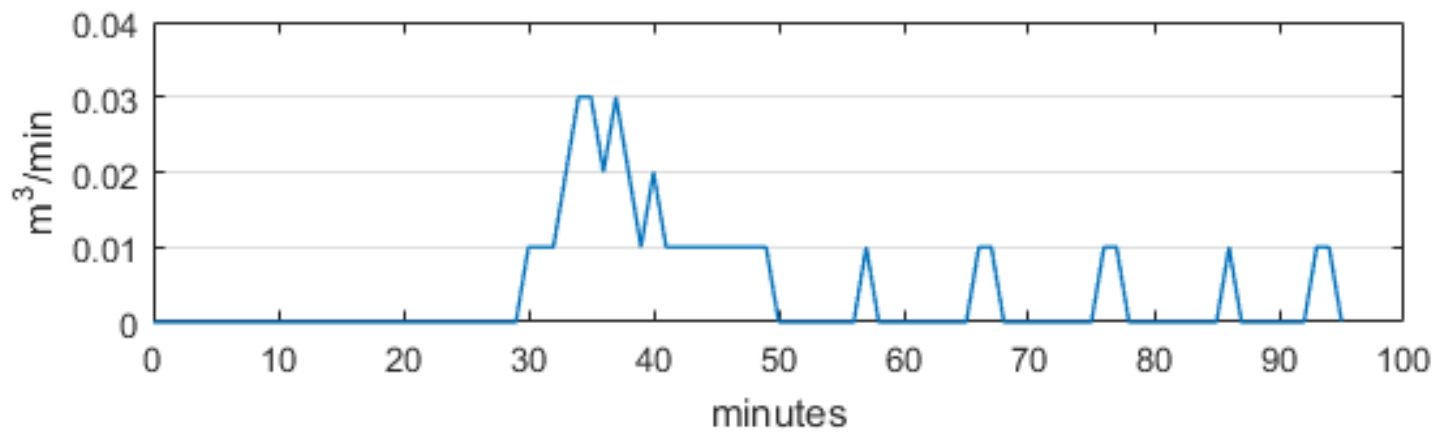

Figure 15: Recorded gas usage during experiment 1

In the second test, while space heating was left running, water heating was triggered for periods of 3 minutes and 2 minutes, with a pause of 10 minutes in between. This resulted in gas usage rising consistently during these periods. By applying the method to the total usage data, the water heating usage is indeed detected and removed in both instances, as shown in Figure 17.

Total Usage

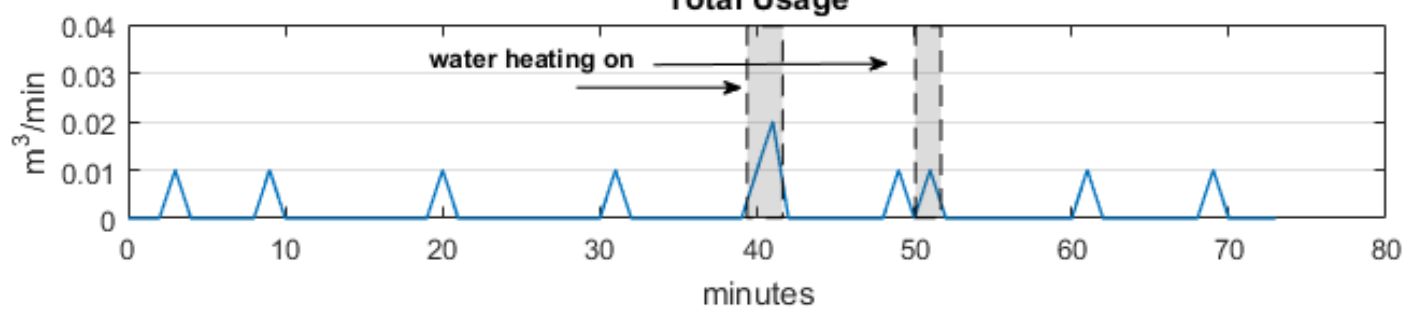

Figure 16 Total gas usage during experiment 2 


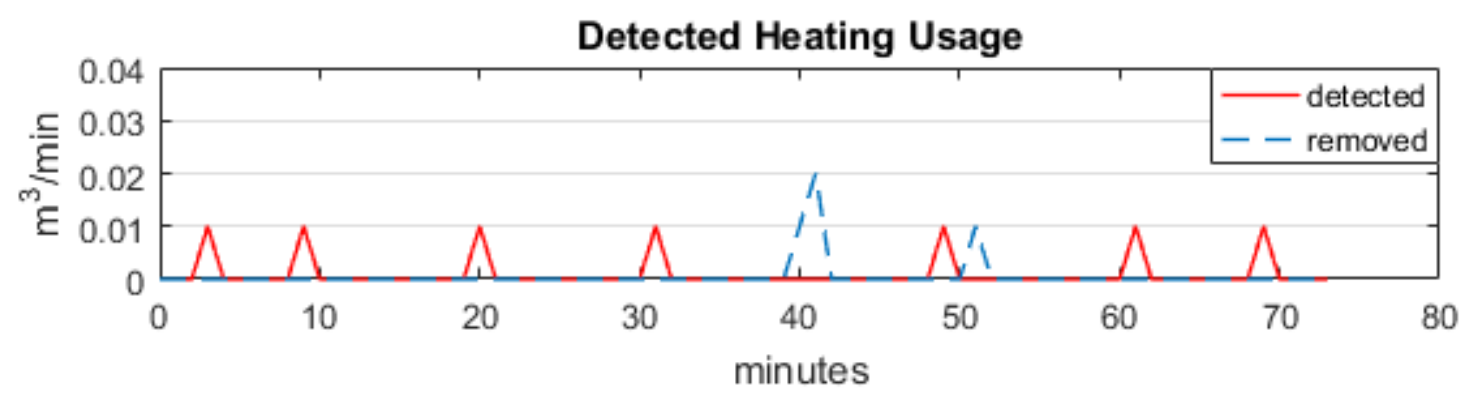

Figure 17 Disaggregated space heating usage during experiment 2

\subsection{Temperature modelling}

Detailed temperature data is available in the studied houses, and the effect of space heating on wholehouse temperature is readily observable and distinguishable from that of other gas uses. This provides a highly suitable opportunity for validation of our method.

The most direct observable effect of space heating is, of course, a significant increase in whole-house internal temperature, whereas gas cooking and hot water heat is localised, and in the case of the latter, a significant portion of it is rapidly lost into the drainage system. Given a model of whole-house temperature change based on gas heating, these differences can be used to validate the results of the method, by comparing the accuracy of temperature predictions using total gas usage data, to those using disaggregated heating usage data. Correct disaggregation should lead to more accurate predictions, as non-heating gas usage would lead to erroneous predictions of temperature rise.

Dynamic modelling methods used to predict thermal response in buildings are numerous and varied, but for the present purpose, an autoregressive (AR) model is well-suited, as it encodes dynamic, short term response, without requiring a tailored model based on the physics of the building. AR models and their derivatives, such as the ARX (eXogenous) model, have been used to predict temperature and energy consumption in buildings in many studies under wildly different conditions in all parts of the world [36] [66], [67] [68].

The process for fitting an appropriate ARX model to energy and temperature data is well known and covered by several sources [69] [66], and is thus dropped here for brevity. The following model is found to be a good fit

$$
T_{i}^{t}=T_{i}^{t-1}+b_{1} \varphi^{t-1}+b_{2} \varphi^{t-2}+\cdots+b_{5} \varphi^{t-5}+c_{1} T_{e}^{t-1}+\varepsilon
$$

Where $T_{i}{ }^{\tau}$ is the average internal temperature at time, $T_{e}{ }^{\tau}$ is the external one, and $\varphi^{\tau}$ is the gas usage during time step $\tau$. Effectively, this model predicts that the temperature at time $t$ will decrease from that at time $t-1$ by an amount linearly proportional to the difference between the internal and external temperatures, while also increasing by an amount linearly proportional to the gas used for heating in the last 5 minutes. The factors are calculated by a statistical fit to the total available data, and the results of model optimisation, in 3 steps, are shown in table 3 below.

\begin{tabular}{|l|l|}
\hline Metric & Value \\
\hline 1-step ahead prediction fit & $97.82 \%$ \\
\hline 5-step ahead prediction fit & $93.94 \%$ \\
\hline Mean Square Error (MSE) & 0.0011 \\
\hline Final Prediction Error (FPE) & 0.0011 \\
\hline
\end{tabular}


607

608

609

610

As the space heating load is the major part of gas usage in the data, the model is tuned to model the response to space heating, with some bias resulting from the presence of non-heating usage. When supplied with the original total gas data, before disaggregation, the results of 1 step-ahead prediction fit the actual measurements closely (94.12\%); however, when a non-heating event occurs, the model erroneously predicts an increase in temperature. Figure 18 shows an example of this.
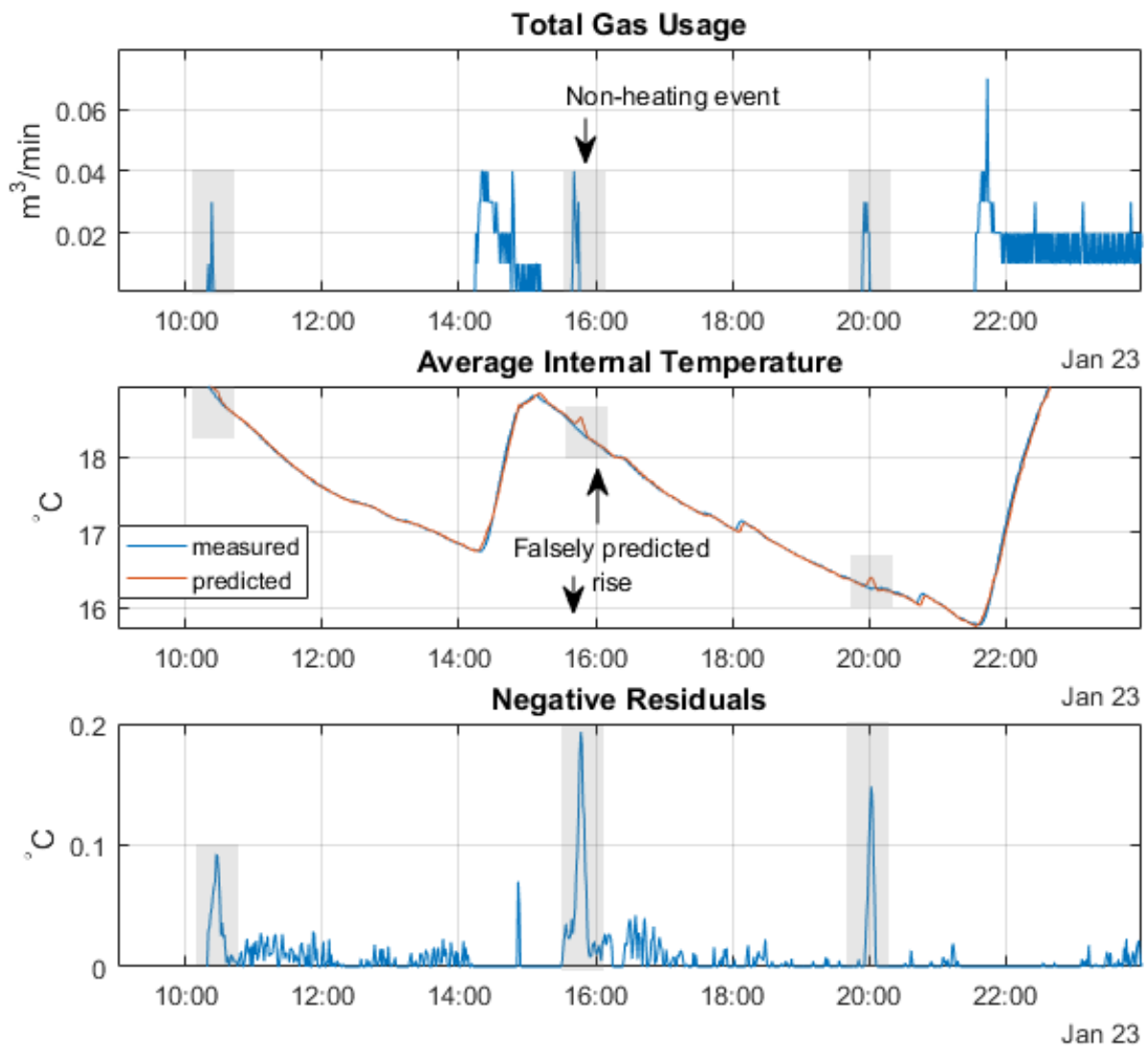

Figure 18 Errors in prediction caused by non-heating events. The last panel only shows negative errors

Using the output of the disaggregation method, the same model was used to predict temperature again, this time with possible non-heating gas usage removed from the input. The results show a significantly better fit to the measured temperature, and a significant decrease in the mean square error, (table 4). A comparison of fit quality before and after disaggregation is given in table 4 below.

\begin{tabular}{|l|c|c|}
\hline Metric & $\begin{array}{c}\text { Value } \\
\text { (total gas) }\end{array}$ & $\begin{array}{c}\text { Value } \\
\text { (disaggregated heating) }\end{array}$ \\
\hline 1-step ahead prediction fit & $94.12 \%$ & $97.82 \%$ \\
\hline 5-step ahead prediction fit & $85.12 \%$ & $93.94 \%$ \\
\hline Mean Square Error (MSE) & 0.0018 & 0.0011 \\
\hline Final Prediction Error (FPE) & 0.0078 & 0.0011 \\
\hline
\end{tabular}

Table 4: Parameters of fit for ARX models, using total and disaggregated heating data

Given that heating is the largest use of gas by a significant margin, these differences are quite significant. This is a clear indication of the validity of the results. The quality of fit of the same model increases 
remarkably after disaggregation, proving that at the very least, the disaggregated series contains significantly less non-heating usage than the original one. Unfortunately, this method cannot be used to detect whether all non-heating usage has been removed, as errors in prediction can stem from other sources, such as unmonitored heat sources (e.g. electric heaters), changes in ventilation (windows, mechanical ventilation, ..etc), and solar gains. For the same reason, using such a model for disaggregation in the first place, though possibly effective, may introduce bias in the results of any analysis based on it, but that question is still open for study.

This confirms the validity of the outputs, particularly as those instances were not easily detectable by sight, but as the experimental data by no means covers all expected circumstances, this is not by itself complete validation, and thus other validation tests are presented below. A more complete validation exercise would be desirable for future work, but is not possible in the context of this study.

\section{Discussion}

Prevailing approaches for investigating the performance gap have had limited success so far in providing the required diagnostic capabilities. At present, there are several challenges to the use of dynamic methods in occupied buildings at the required scale, but data collection remains a key obstacle for research and actual implementation, which has led to increased interest in simpler methods that lack accuracy and do not provide the diagnostic insight required for tackling the gap.

The presented method improves the potential applicability of dynamic analysis methods to existing occupied residential buildings by making dependence on smart meter data an option for heating energy measurement even when other uses are present. This reduces the potential cost greatly, as any alternative requiring the installation of extra instrumentation would entail the cost of the hardware and the labour and time of skilled technicians; whereas this method only requires the addition of some processing step to the smart metering infrastructure. This can take the shape of a small program running on the same device being used as an inhome monitor, which cuts down the cost of deployment to those of a software update for the existing monitors. There are already calls for smart metering standards and commercial devices to incorporate the ability to host different software and forms of feedback, rather than simply rely on the software provided by the utility company [66], this could pave the way to a different service model for employing smart meter data, in which service providers can create applications that the consumers can give access to their data to, similar to the Green Button Initiative model introduced in the US [67].

The result of applying the method to both datasets is a time series containing only gas usage matching the identified pattern of space heating usage, and free from any anomalous spikes. The effect of these events on the internal temperature matches predictions based on an optimised dynamic model to a high level, indicating that the results are likely accurate. An experimental validation exercise was also carried out with positive results. Though this may not be sufficient to measure the performance of our implementation in a precise fashion, it does indicate that this method, or ones based on it, are a successful approach to isolating heating gas usage without resorting to any additional data, which makes the use of smart meter data for a dynamic model of energy performance possible at high frequencies with a significantly reduced uncertainty, and effectively removes the need for the cumbersome instrumentation setups that have been required so far, in cases where meter readings are sufficiently accurate.

Regarding general applicability, the observed characteristics of the heat series seem to stem mostly from the fact that the boiler generating is it a combi boiler, which may give cause to limit the intended domain of application to this type of boilers, but the method is general enough to be adaptable to different types, as long as their behaviour while heating hot water (likely in a cylinder) is distinguishable from that of space 
heating. In the available data, we see that although hot water heating did occur in the form of spikes most of the time, longer loads (baths) produce different patterns. Additionally, hot water spikes were effectively identical to those observed as parts of the heating cycle, which points to a strength of this method; its reliance on dynamic variation prevents otherwise confounding behaviours from causing false detection. Nonetheless, it is still necessary to test on different data sources to validate and improve it, as several factors may be less than ideal in other setups. The window detection method, in particular, though seeming reasonable for any signature, may be more error-prone with different forms of heating control or with an increased number of gas-powered systems. The nature of operation in House 2 is already challenging to capture with without careful tuning, but a more sophisticated filter would likely manage to resolve this issue. This is not likely to have an impact on the applicability of the method in general, as heating events are expected to be the most pronounced instances of sustained use in any gas usage time series; but for the sake of designing an automatic optimisation scheme that is applicable in all cases, a method with more configurable parameters may yield more precise results. Though we have suggested several optimisation options for automating parameter selection, this is not a problem that can generally be solved confidently with such a limited dataset.

Nonetheless, given the low number of configurable operations, an automated robust implementation seems within reach. This can be realised through possible future development of this method to include automatic detection of signatures based on clustering, which has been demonstrated in various applications before, including disaggregation and energy signatures $[68,69,70]$. It is possible that a set of simple heuristics aided by a clustering algorithm could find heating signatures automatically and reliably, which could in turn prove important for datasets captured at a lower sampling frequency where patterns would be less well-defined, resulting in a reduced requirement for sampling frequency.

Since reliability of disaggregation seems to be good, there is indeed potential for gas disaggregation to become part of the official smart meter infrastructure. In practice, a universal deployment might take the form of a software component running locally on smart-meter processors or on existing home energy monitors, in order to make use of 1-minute (or less) frequency data without burdening the network with a large amount of readings.

\section{Conclusion}

A method for disaggregating space heating demand from total gas meter readings is presented, developed and tested using data from a case study in two houses in the south west of England. It is found that the profile of use for the installed gas-fired heating systems is distinct and consistent; easily identified in the complete time series. Automatically detecting all possible windows of heating activity and comparing them to an identified signature was found to produce credible results, supported by the available evaluation methods. Though the method is intended to be quite generic, further study is recommended with different types of boilers and heating systems in various contexts, to ensure robustness and enable the creation of an automated optimisation routine to automatically detect heating signatures and tune selection and filtering parameters.

The success of the presented method in achieving the required separation between the gas load of the heating system and that of other appliances could allow studies of building performance evaluation using smart-meter data in houses where heating is provided by gas. Analysis using dynamic models with this data is possible, should the other required inputs be available, and is expected to be of much higher use for diagnosing performance gaps than typical steady state ones.

\section{Acknowledgment}

This work has been generously funded by the British Council Newton Fund, as part of the Institutional Links program, award reference 172732496.

\section{Declaration of interest}


The authors have no financial or personal relationships with any individual or organisation, or any other interests that could inappropriately influence this work. The funding body financing this work had no influence on its design, execution, or decisions relating to publishing.

[1] M. Economidou, B. Atanasiu, C. Despret, J. Maio, I. Nolte, O. Rapf and others, "Europe's buildings under the microscope. A country-by-country review of the energy performance of buildings," Buildings Performance Institute Europe (BPIE), 2011.

[2] M. Maivel, K. Kuusk, R. Simson, J. Kurnitski, T. Kalamees and others, "Status on the Ground-Overview of existing surveys on energy performance related quality and compliance," QUALICHeCK Project Report, 2015.

[3] P. De Wilde, "The gap between predicted and measured energy performance of buildings: A framework for investigation," Automation in Construction, vol. 41, pp. 40-49, 2014.

[4] E. Mangematin, G. Pandraud and D. Roux, "Quick measurements of energy efficiency of buildings," Comptes Rendus Physique, vol. 13, pp. 383-390, 2012.

[5] P. Boisson and R. Bouchié, "ISABELE method: In-Situ Assessment of the Building EnveLope pErformances," in 9th International Conference on System Simulation in Buildings, 2014.

[6] J. K. Kissock, J. S. Haberl and D. E. Claridge, "Inverse modeling toolkit: numerical algorithms (RP-1050)," Transactions-American society of heating refrigerating and air conditioning engineers, vol. 109, pp. 425434, 2003.

[7] M. F. Fels, "PRISM: an introduction," Energy and Buildings, vol. 9, pp. 5-18, 1986.

[8] R. Jack, Building diagnostics: practical measurement of the fabric thermal performance of houses, PhD Thesis, PhD, University of Loughborough, 2015.

[9] J. Berger, S. T. Tasca-Guernouti and M. Humbert, "Experimental method to determine the energy envelope performance of buildings," 2010.

[10] D. Butler and A. Dengel, Review of Co-heating Test Methodologies: Primary Research, NHBC Foundation, 2013.

[11] S. Hammarsten, "A critical appraisal of energy-signature models," Applied Energy, vol. 26, pp. 97-110, 1987.

[12] R. Everett, "Rapid thermal calibration of houses report ERG055," Science and Engineering Research Council Report. London, 1985.

[13] C. P. Underwood and F. W. H. Yik, "Modelling Heat Transfer in Building Envelopes," in Modelling Methods for Energy in Buildings, Blackwell Publishing Ltd, 2008, pp. 47-92. 
[14] G. Bauwens, In Situ Testing of a Building's Overall Heat Loss Coefficient-Embedding Quasi-stationary and Dynamic Tests in a Building Physical and Statistical Framework, 2015.

[15] J. J. Bloem, System identification applied to building performance data, Office for Official Publications of the European Communities, 1994.

[16] R. R. Crawford and J. E. Woods, "A method for deriving a dynamic system model from actual building performance data," ASHRAE Trans.;(United States), vol. 91, 1985.

[17] A. Afram and F. Janabi-Sharifi, "Theory and applications of HVAC control systems - A review of model predictive control (MPC)," Building and Environment, vol. 72, pp. 343-355, 2014.

[18] M. D. Knudsen and S. Petersen, "Demand response potential of model predictive control of space heating based on price and carbon dioxide intensity signals," Energy and Buildings, vol. 125, pp. 196204, 2016.

[19] H. Madsen, P. Bacher, G. Bauwens, A.-H. Deconinck, G. Reynders, S. Roels, E. Himpe and G. Leth\{\'e\}, "Thermal Performance Characterization using Time Series Data-IEA EBC Annex 58 Guidelines," International Energy Agency, 2015.

[20] H. Harb, N. Boyanov, L. Hernandez, R. Streblow and D. Müller, "Development and validation of grey-box models for forecasting the thermal response of occupied buildings," Energy and Buildings, vol. 117, pp. 199-207, 2016.

[21] P. A. Strachan and L. Vandaele, "Case studies of outdoor testing and analysis of building components," Building and Environment, vol. 43, pp. 129-142, 2008.

[22] P. R. Armstrong, J. A. Dirks, R. W. Reilly, J. W. Currie, R. J. Nesse, B. Nekrasov and O. V. Komarov, "Russian apartment building thermal response models for retrofit selection and verification," ACEEE Summer Study on Energy Efficiency in Buildings, Monterey, CA, 2000.

[23] V. Dimitriou, S. K. Firth, T. M. Hassan, T. Kane and F. Fouchal, Developing suitable thermal models for domestic buildings with Smart Home equipment, The Bartlett, UCL Faculty of the Built Environment Institute for Environmental Design and Engineering (C IBPSA-England), 2014.

[24] W. Swan, R. Fitton and P. Brown, "A UK practitioner view of domestic energy performance measurement," Proceedings of the Institution of Civil Engineers - Engineering Sustainability, vol. 168, pp. 140-147, 2015.

[25] D. Connolly, "Heat Roadmap Europe: Quantitative comparison between the electricity, heating, and cooling sectors for different European countries," Energy, vol. 139, pp. 580-593, 2017.

[26] S. R. Vitullo, Disaggregating time series data for energy consumption by aggregate and individual customer, PhD Thesis, Marquette University, 2011.

[27] M. Wytock and J. Z. Kolter, "Contextually Supervised Source Separation with Application to Energy Disaggregation.," in $A A A l, 2014$.

[28] A. Zoha, A. Gluhak, M. A. Imran and S. Rajasegarar, "Non-intrusive load monitoring approaches for 
disaggregated energy sensing: A survey," Sensors, vol. 12, pp. 16838-16866, 2012.

[29] S. R. Vitullo, R. H. Brown, G. F. Corliss and B. M. Marx, "Mathematical models for natural gas forecasting," Canadian applied mathematics quarterly, vol. 17, pp. 807-827, 2009.

[30] S. Yamagami, H. Nakamura and A. Meier, "Non-Intrusive Submetering of Residential Gas Appliances," in Proceedings of the ACEEE Summer Study on Energy Efficiency in Buildings, 1996.

[31] R. Sonderegger, "Diagnostic tests determining the thermal response of a house," Lawrence Berkeley National Laboratory, 2010.

[32] A. Rabl, "Parameter estimation in buildings: Methods for dynamic analysis of measured energy use," J. Sol. Energy Eng.;(United States), vol. 110, 1988.

[33] P. Bacher, P. A. Saint-Aubain, L. E. Christiansen and H. Madsen, "Non-parametric method for separating domestic hot water heating spikes and space heating," Energy and Buildings, vol. 130, pp. 107-112, 2016.

[34] K. K. Andersen, H. Madsen and L. H. Hansen, "Modelling the heat dynamics of a building using stochastic differential equations," Energy and Buildings, vol. 31, pp. 13-24, 2000.

[35] M. Killian and M. Kozek, "Ten questions concerning model predictive control for energy efficient buildings," Building and Environment, vol. 105, pp. 403-412, 2016.

[36] G. Mustafaraj, G. Lowry and J. Chen, "Prediction of room temperature and relative humidity by autoregressive linear and nonlinear neural network models for an open office," Energy and Buildings, vol. 43, pp. 1452-1460, 2011.

[37] R. BOUCHIE, F. Alzetto, A. Brun, C. Weeks, M. Preece, A. Muhammad and M. Sisinni, "Methodologies for the Assessment of Intrinsic Energy Performance of Buildings Envelope," March 2015. [Online]. Available: http://performerproject.eu/wp-content/uploads/2015/04/PERFORMER_D12_Methodologies-for-the-Assessment-of-Intrinsic-Energy-Performance-of-Buildings-Envelope.pdf. [Accessed 15 May 2018].

[38] P. Vigo and F. Cascetta, "Sensors for measuring flow," Sensors Set: A Comprehensive Survey, pp. 373436, 1993.

[39] G. Ficco, "Metrological performance of diaphragm gas meters in distribution networks," Flow Measurement and Instrumentation, vol. 37, pp. 65-72, 2014.

[40] G. Buonanno, "On field characterisation of static domestic gas flowmeters," Measurement, vol. 27, pp. 277-285, 2000.

[41] H. Kershaw, T. Lelyveld, S. Burton, G. Orr, H. Charlick, T. Dennish and M. Crowther, "In-situ monitoring of efficiencies of condensing boilers-TPI control project extension," Energy Saving Trust and Department of Energy |\& Climate Change, 2010.

[42] B. Sodagar and D. Starkey, "The monitored performance of four social houses certified to the Code for Sustainable Homes Level 5," Energy and Buildings, vol. 110, pp. 245-256, 2016. 
[43] A. Beizaee, D. Allinson, K. J. Lomas, E. Foda and D. L. Loveday, "Measuring the potential of zonal space heating controls to reduce energy use in UK homes: The case of un-furbished 1930s dwellings," Energy and Buildings, vol. 92, pp. 29-44, 2015.

[44] M. Zeifman and K. Roth, "Non-Intrusive Appliance Load Monitoring (NIALM): Review and Outlook," IEEE Transactions on Consumer Electronics, vol. 75, no. 1, pp. 76-84, 2011.

[45] S. R. Shaw, S. B. Leeb, L. K. Norford and R. W. Cox, "Nonintrusive Load Monitoring and Diagnostics in Power Systems," IEEE Transactions on Instrumentation and Measurement, vol. 57, pp. 1445-1454, 7 2008.

[46] G. W. Hart, "Nonintrusive appliance load monitoring," Proceedings of the IEEE, vol. 80, pp. 1870-1891, 1992.

[47] V. Stankovic, J. Liao and L. Stankovic, "A graph-based signal processing approach for low-rate energy disaggregation," in 2014 IEEE Symposium on Computational Intelligence for Engineering Solutions (CIES) Proceedings, 2014, pp. 81-87.

[48] Kamstrup, "MULTICAL 403: Specifications," Kamstrup, 2017. [Online]. Available: https://www.kamstrup.com/en-en/products-solutions/thermal-energy-meters/multical-403. [Accessed 15 May 2018].

[49] E. Instromet, "BK-G40 Product Specifications," 11 2017. [Online]. Available: https://www.elsterinstromet.com/de/product-details/165/de/BK-G40,_BK-G65,_BK-G100. [Accessed 15 May 2018].

[50] M. Baranski and J. Voss, "Genetic algorithm for pattern detection in NIALM systems," in Systems, man and cybernetics, 2004 ieee international conference on, 2004.

[51] K. A. Nguyen, R. A. Stewart and H. Zhang, "An Intelligent Pattern Recognition Model to Automate the Categorisation of Residential Water End-use Events," Environ. Model. Softw., vol. 47, pp. 108-127, 9 2013.

[52] E. Thomaz, V. Bettadapura, G. Reyes, M. Sandesh, G. Schindler, T. PI\{\"o\}tz, G. D. Abowd and I. Essa, "Recognizing water-based activities in the home through infrastructure-mediated sensing," in Proceedings of the 2012 ACM Conference on Ubiquitous Computing, 2012.

[53] M. Tewolde, J. P. Longtin, S. R. Das and S. Sharma, "Determining appliance energy usage with a highresolution metering system for residential natural gas meters," Applied Energy, vol. 108, pp. 363-372, 2013.

[54] G. Cohn, S. Gupta, J. Froehlich, E. Larson and S. N. Patel, “GasSense: Appliance-level, single-point sensing of gas activity in the home," in International Conference on Pervasive Computing, 2010.

[55] G. M. Huebner, J. Cooper and K. Jones, "Domestic energy consumption-What role do comfort, habit, and knowledge about the heating system play?," Energy and Buildings, vol. 66, pp. 626-636, 2013.

[56] E. Keogh and S. Kasetty, "On the need for time series data mining benchmarks: a survey and empirical demonstration," Data Mining and knowledge discovery, vol. 7, pp. 349-371, 2003. 
[57] D. J. Berndt and J. Clifford, "Using dynamic time warping to find patterns in time series.," in KDD workshop, 1994.

[58] B.-H. Juang, "On the hidden Markov model and dynamic time warping for speech recognition-A unified view," Bell Labs Technical Journal, vol. 63, pp. 1213-1243, 1984.

[59] J. Aach and G. M. Church, "Aligning gene expression time series with time warping algorithms," Bioinformatics, vol. 17, pp. 495-508, 2001.

[60] E. G. Caiani, A. Porta, G. Baselli, M. Turiel, S. Muzzupappa, F. Pieruzzi, C. Crema, A. Malliani and S. Cerutti, "Warped-average template technique to track on a cycle-by-cycle basis the cardiac filling phases on left ventricular volume," in Computers in Cardiology 1998, 1998.

[61] G. Elafoudi, L. Stankovic and V. Stankovic, "Power disaggregation of domestic smart meter readings using dynamic time warping," in 6th International Symposium on Communications, Control and Signal Processing (ISCCSP), 2014, IEEE, 2014.

[62] E. Keogh and C. A. Ratanamahatana, "Exact indexing of dynamic time warping," Knowledge and Information Systems, vol. 7, pp. 358-386, 0132005.

[63] V. Chandola, A. Banerjee and V. Kumar, "Anomaly Detection: A Survey," ACM Comput. Surv., vol. 41, pp. 15:1--15:58, 72009.

[64] W. K. Lee, G. S. K. Fung, H. Y. Lam, F. H. Y. Chan and M. Lucente, "Exploration on load signatures," in International conference on electrical Engineering (ICEE), 2004.

[65] V. Chandola, A. Banerjee and V. Kumar, "Anomaly detection for discrete sequences: A survey," IEEE Transactions on Knowledge and Data Engineering, vol. 24, pp. 823-839, 2012.

[66] M. Pullinger, H. Lovell and J. Webb, "Influencing household energy practices: a critical review of UK smart metering standards and commercial feedback devices," Technology Analysis |\& Strategic Management, vol. 26, pp. 1144-1162, 2014.

[67] D. S. Sayogo and T. A. Pardo, "Understanding Smart Data Disclosure Policy Success: The Case of Green Button," in Proceedings of the 14th Annual International Conference on Digital Government Research, New York, NY, USA, 2013.

[68] D. Minnen, T. Starner, I. Essa and C. Isbell, "Discovering characteristic actions from on-body sensor data," in Wearable computers, 2006 10th IEEE international symposium on, 2006.

[69] F. Gullo, G. Ponti, A. Tagarelli, S. M. Ruffolo and D. Labate, "Low-voltage Electricity Customer Profiling Based on Load Data Clustering," in Proceedings of the 2009 International Database Engineering |\&|\#38; Applications Symposium, New York, NY, USA, 2009.

[70] E. Himpe and A. Janssens, "Data-Driven Modelling of the Energy Use in Dwellings using Smart Meter Data," in CLIMA 2016-12th REHVA World Congress, 2016. 
Defined as the rate of heat loss (W) from the entire envelope of a building per Kelvin of temperature difference between the internal and external environment. 